\title{
Peripheral Production and Decay of Resonances in High-Energy Meson-Nucleon Collisions*
}

\author{
J. D. Jackson, $\dagger$ and J. T. Donohue \\ Department of Physics, University of Illinois, Urbana, Illinois \\ AND \\ K. GotTFrIED $\ddagger$ \\ Laboratory of Nuclear Studies, Cornell University, Ithaca, New York \\ AND \\ R. Keyser and B. E. Y. Svensson \\ CERN, Geneva, Switzerland \\ (Received 5 March 1965)
}

\begin{abstract}
Data on quasi-two-body reactions initiated by high-energy meson-nucleon collisions are analyzed from the viewpoint of the peripheral model. The basic interaction mechanism is assumed to be pseudoscalar and/or vector exchange, and absorptive effects arising from coupling between different channels are taken into account. Excellent agreement is found between the one-pion-exchange model and the production and decay data for the processes $\pi^{ \pm} p \rightarrow \rho^{ \pm} p$ for incident momenta from $\sim 2$ to $8 \mathrm{GeV} / c$. A unified analysis of the reactions $K^{ \pm} p \rightarrow K^{* \pm} p$ at $3 \mathrm{GeV} / c$, and $K^{+} n \rightarrow K^{0} p$ at $2.3 \mathrm{GeV} / c$, is presented; a single pair of vector coupling constants is able to account for the various angular distributions of production. The theory predicts, and preliminary data confirm, appreciable momentum-transfer dependence of the $K^{*}$ spin-density matrix. Equally satisfactory fits to the data are found for $K^{+} p \rightarrow K^{0} N^{*++}$ at $3 \mathrm{GeV} / c, K^{-} p \rightarrow \pi^{-} Y^{*}$ (1385) at $2.24 \mathrm{GeV} / c$, and $\pi^{+} n \rightarrow \omega p$ at $3.25 \mathrm{GeV} / c$. In the latter reaction the absorption model reproduces the large departure of the $\omega$-decay correlations from those characteristic of $\rho$ exchange in the absence of absorption. The model cannot explain why the differential cross section for $\pi^{+} p \rightarrow \pi^{0} N^{*}$ is considerably narrower than for $K^{+} p \rightarrow K^{0} N^{*}$. The theoretical momentum-transfer distributions and decay correlations for double resonance production $\left(\pi p \rightarrow \rho N^{*}, K p \rightarrow K^{*} N^{*}\right)$ are in general agreement with the data, as are the results on combined decay correlations of $\rho$ and $N^{*}$ in $\pi^{+} p \rightarrow \rho^{0} N^{*}$. But the calculated absolute cross sections are considerably larger than observed, probably because the model fails to include the requirements of unitarity, important when the couplings are large. The determination of vector-meson coupling constants is discussed. Because of fundamental difficulties associated with vector-meson exchange amplitudes at high energies, it is concluded that important refinements of the model are necessary if such coupling constants are to be inferred from the data.
\end{abstract}

\section{INTRODUCTION}

$\mathrm{T}$ HE importance of absorptive effects due to competition from other inelastic channels in peripheral production processes has recently received considerable attention. ${ }^{1-7}$ In a previous paper, ${ }^{5}$ referred to henceforth as I, we presented a formulation which incor-

* Partially supported by the U. S. Office of Naval Research, under Contract No. ONR 1834 (05) at Illinois, and Contract No. ONR 401 (51) at Cornell.

$\dagger$ Ford Foundation Fellow at CERN, 1963-64, during which time this work was begun:

† Supported in part during $1963-64$ by the John Simon Guggenheim Memorial Foundation and the Ford Foundation.

${ }_{1}^{1}$ N. J. Sopkovich, Nuovo Cimento 26, 186 (1962).

2 A. Dar, M. Kugler, Y. Dothan, and S. Nussinov, Phys. Rev. Letters 12, 82 (1964); A. Dar and W. Tobocman, ibid. 12, 511 (1964); A. Dar, ibid. 13, 91 (1964).

${ }_{3}$ L. Durand and Y. T. Chiu, Phys. Rev. Letters 12, 399 (1964);

13, 45(E) (1964); L. Durand, Proceedings of the Conference on Particle and High-Energy Physics, Boulder, Colorado, 1964 (to be published); L. Durand and Y. T. Chiu, Phys. Rev. 137, B1530 (1965).

${ }^{4}$ M. H. Ross and G. L. Shaw, Phys. Rev. Letters 12, 627 (1964); R. C. Arnold, Phys. Rev. 136, B1388 (1964).

${ }_{5} \mathrm{~K}$. Gottfried and J. D. Jackson, Nuovo Cimento 34, 735 (1964).

${ }^{6} \mathrm{~K}$. Gottfried, J. D. Jackson, and B. E. Y. Svensson, in Proceedings of the 12th Annual International Conference on HighEnergy Physics, Dubna, 1964 (Moscow, 1965).

${ }^{7}$ J. D. Jackson, Rev. Mod. Phys. 37, 484 (1965). porates these absorptive effects in a simple phenomenological fashion. This method was there applied to the reaction $\pi^{-} p \rightarrow \rho^{-} p$ at $4 \mathrm{GeV} / c$; excellent agreement with the experimental angular distribution of production was achieved, and the theoretical decay correlation of the $\rho$ meson was found to be consistent with the meager data then available.

In this paper we report the results of similar calculations for a number of different reactions over a rather broad range of incident momenta. To be specific, we shall quote results for the quasi-two-body final states $\rho N, \pi N^{*}, \rho N^{*}$, and $\omega N$ produced in $\pi N$ collisions between 1.6 and $8 \mathrm{GeV} / c$, as well as the configurations $K^{*} N, \bar{K}^{*} N, K N^{*}, \pi Y^{*}$, and $K^{*} N^{*}$ arising from $K N$ and $\bar{K} N$ encounters from 2 to $5 \mathrm{GeV} / c$.

Even though our calculations are not exhaustive, they suffice to delineate roughly the domain of applicability of the modified one-particle-exchange model. This domain of validity appears to be rather limited. Thus, on the one hand, the excellent agreement found for the reaction $\pi p \rightarrow \rho p$ at $4 \mathrm{GeV} / c$ reported in $\mathrm{I}$ is extended with equal success to the entire momentum range of 1.6 to $8 \mathrm{GeV} / c$. Similarly, in the more limited momentum interval of $2-3 \mathrm{GeV} / c$, the model successfully describes the reactions $K N \rightarrow K^{*} N, \bar{K} p \rightarrow \bar{K}^{*} p$ 
and $K p \rightarrow K^{0} N^{*}$. On the other hand, to the extent that the elastic and total cross sections are relatively slowly varying functions of energy, the absorptive effects do not prevent the familiar increase with energy of vectorand higher spin-exchange amplitudes. This emphasizes that the recipe for incorporating the effects of coupled channels used by us is not sufficiently sophisticated to provide a model valid in the high-energy limit. It is of course well known that Regge-pole exchange will give production amplitudes consistent with unitarity in the high-energy limit. But the generation of Regge-pole behavior from the multiperipheral model ${ }^{8}$ indicates a connection between the notion of Regge exchange and the absorptive model. The simultaneous use of Reggepole exchange and absorption inferred from elastic scattering is therefore inconsistent. On the other hand, at the energies considered here the simple Regge-pole exchange mechanism is of doubtful validity.

The calculations presented here follow closely the format set out in I. But minor improvements in the calculation have been made, necessitating a recapitulation of the essential formulas. Let $\left\langle\lambda_{c} \lambda_{d}\left|B_{j}\right| \lambda_{a} \lambda_{b}\right\rangle$ be the one-particle-exchange amplitude for the partial wave of angular momentum $j$, the helicities of the particles in the initial and final states being $\left(\lambda_{a}, \lambda_{b}\right)$ and $\left(\lambda_{c}, \lambda_{d}\right)$, respectively. In the high-energy limit, and when the range of the exchange interaction is short compared to that of the absorptive interactions which give rise to the elastic scattering in the initial and final states, the distorted-wave Born amplitude is (see I-3.9)

$$
\left\langle\lambda_{c} \lambda_{d}\left|T_{j}\right| \lambda_{a} \lambda_{b}\right\rangle=e^{i \delta_{j}^{(-)}}\left\langle\lambda_{c} \lambda_{d}\left|B_{j}\right| \lambda_{a} \lambda_{b}\right\rangle e^{i \delta_{j}(+)},
$$

where $\delta_{j} j^{(-)}$and $\delta_{j}{ }^{(+)}$are the complex elastic-scattering phase shifts in the final and initial channels, respectively. [In I we used the notation $\frac{1}{2} \chi^{( \pm)}=\delta^{( \pm)}$.] The full helicity amplitude is then

$$
\begin{aligned}
\left\langle\lambda_{c} \lambda_{d}|M| \lambda_{a} \lambda_{b}\right\rangle= & \sum_{j=j_{0}}^{\infty}\left(j+\frac{1}{2}\right) e^{i \delta_{j}(-)} \\
& \times\left\langle\lambda_{c} \lambda_{d}\left|B_{j}\right| \lambda_{a} \lambda_{b}\right\rangle e^{i \delta_{j}(+)} J_{n}\left(\left(j+\frac{1}{2}\right) \omega\right),
\end{aligned}
$$

where $\omega=2 \sin (\theta / 2), n=\left(\lambda_{c}-\lambda_{d}\right)-\left(\lambda_{a}-\lambda_{b}\right)$, and $j_{0}$ is the larger of the two numbers $\left|\lambda_{a}-\lambda_{b}\right|$ and $\left|\lambda_{c}-\lambda_{d}\right|$. In (2) we have approximated the rotational function by the appropriate Bessel function [see (I-3.6)].

In I we converted (2) into an integral over the variable $x=j+\frac{1}{2}$, with $x=0$ as the lower limit of integration. The error introduced by retaining the unphysical partial waves with $j<j_{0}$ vanishes in the high-energy limit. This approximation is therefore consistent with the others already implicit in (2). On the other hand, the presence of these unphysical angular-momentum states leads to spurious violations of the unitarity bound

${ }^{8}$ D. Amati, A. Stanghellini, and S. Fubini, Nuovo Cimento 26 , 896 (1962); D. Amati, M. Cini, and A. Stanghellini, ibid. 30, 193 (1963). [cf. (I-3.13)] as $x \rightarrow 0$, even when absorption is included. This effect does not occur in $\rho$ production, but becomes important in reactions such as $K N \rightarrow K^{*} N^{*}$ where amplitudes with $n>2$ enter significantly. In the calculation reported here we have therefore put the proper lower limit into the $x$ integrations, namely $j_{0} \leq x<\infty$.

In I the elastic amplitudes were approximated by a Gaussian function of impact parameter:

Here

$$
e^{2 i j_{j}^{( \pm)}} \simeq 1-C_{ \pm} e^{-\gamma_{ \pm} x^{2}}
$$

$$
\begin{aligned}
& C_{+}=\sigma_{T}^{(+)} / 4 \pi A_{+}, \\
& \gamma_{+}=1 / 2 q^{2} A_{+}, \text {etc. }
\end{aligned}
$$

where $\sigma_{T}{ }^{(+)}$is the total cross section for $a-b$ scattering, $q$ is the incident-center-of-mass momentum, and $A_{+}$is the parameter which appears in the elastic angular distribution [i.e., $d \sigma_{\mathrm{el}} / d t \propto e^{A t}$, cf. (I-2.11)]; analogous definitions apply to the final state quantities $C_{-}$and $\gamma_{-}$. In using (3) we tacitly assumed that the absorptivity is a function of the total angular momentum $j$, whereas it is probably more reasonable to assume that the most relevant variable is the orbital angular momentum. A treatment of the absorptive effects in terms of orbital angular-momentum states cannot be expected to lead to qualitatively different results from those obtained when the depletion of the incoming and outgoing waves is related to the total angular momentum. In view of our very rudimentary understanding of the various interactions involved, refinements which do not lead to significant alteration of the final results are not justifiable unless they do not complicate the calculation procedures. We therefore continue to express all amplitudes in terms of the variable $j$ (or equivalently, $x$ ). We have, however, made one slight alteration; namely, we now use

$$
e^{2 i \delta_{j}^{( \pm)}} \rightarrow 1-C_{ \pm} e^{-\gamma_{ \pm}(x-1 / 2)^{2}}
$$

instead of (3). This form has the property that when $C_{+}=1$ (and/or $C_{-}=1$ ) the lowest partial wave contributing to amplitudes with $j_{0}=\frac{1}{2}$ is completely absorbed. Roughly speaking, this is equivalent to total absorption in relative $S$ states.

In carrying out the partial-wave analysis of the Born amplitude we shall henceforth use the approximate form

$$
\frac{\omega^{n}}{\epsilon^{2}+\omega^{2}} \simeq \epsilon^{n} \int_{j_{0}}^{\infty} K_{n}(\epsilon x) J_{n}(\omega x) x d x,
$$

instead of the exact form (I-4.11) in which the lower limit of integration is zero. The error incurred here is $\sim\left(\epsilon^{2}+\omega^{2}\right) j_{0}^{2}$. For small scattering angles such that $\omega \lesssim \epsilon$, the error is thus of order $\epsilon^{2} j_{0}^{2}$, which vanishes in the high-energy limit since $\epsilon^{2} \simeq m_{x}^{2} / s$, where $m_{x}$ is the

\footnotetext{
${ }^{9}$ Here we always have in mind collisions between nucleons and spinless mesons; otherwise $j_{0}$ could be zero.
} 
mass of the exchanged particle. The final form for the amplitude is therefore

$$
\begin{aligned}
M_{n}{ }^{(k)}=A_{n}{ }^{(k)} & \int_{j_{0}}^{\infty} J_{n}(\omega x) K_{n}(\epsilon x)\left[1-C_{+} e^{-\gamma_{+}(x-1 / 2)^{2}}\right]^{1 / 2} \\
& \times\left[1-C_{-} e^{-\gamma_{-}(x-1 / 2)^{2}}\right]^{1 / 2} x d x \\
+ & \bar{A}_{n}{ }^{(k)}\left[1-C_{+} e^{-\gamma_{+}\left(j_{0}-1 / 2\right)^{2}}\right]^{1 / 2} \\
& \quad \times\left[1-C_{-} e^{-\gamma_{-}\left(j_{0}-1 / 2\right)^{2}}\right]^{1 / 2} d_{\lambda \mu}{ }^{j_{0}}(\vartheta)
\end{aligned}
$$

where all symbols not yet defined are the same as in (I-4.14).

We should like to stress that the refinements in the formulation of I which have just been described do not alter the theoretical predictions in a qualitative fashion, even though they sometimes lead to quite noticeable changes in the numerical values of cross sections and decay correlations. Moreover, the more glaring failures of the model (such as the energy dependence of vectorexchange amplitudes) cannot be ascribed to these rather minor ambiguities, nor to the mathematical approximations which we have made. When and where the model fails, it presumably does so because it does not adequately describe the dynamics governing the process in question.

\section{THE REACTION $\pi p \rightarrow \varrho p$}

Experimental data on this reaction are available from threshold to $8 \mathrm{GeV} / c^{10-17}$ Before we compare the theoretical predictions with these data, we shall discuss briefly the sensitivity of the results to the parameters which appear in the model.

\section{A. Dependence on Mass of Exchanged Particle}

In I we found that absorptive effects lead to a drastic forward peaking of the differential cross section. This raises the question of whether the angular distribution

${ }^{10} \mathrm{H}$. Foelsche, E. C. Fowler, H. L. Kraybill, J. R. Sanford, and D. Stonehill, Proceedings of the 1962 Annual International Conference on High-Energy Nuclear Physics at CERN, edited by J. Prentki (CERN, Geneva, 1962), p. 36: $\pi^{+} p$ near $1 \mathrm{GeV} / c$.

${ }^{11}$ Saclay-Orsay-Bari-Bologna Collaboration, Nuovo Cimento 29, 515 (1963): $\pi^{-} p$ at $1.6 \mathrm{GeV} / c$.

${ }^{12}$ Saclay-Orsay-Bari-Bologna Collaboration, Proceedings of the Sienna International Conference on Elementary Particles, 1963, edited by G. Bernadini and G. P. Puppi (Società Italiana di Fisica, Bologna, 1963) Vol. I, p. 239; also unpublished report, June, $1964: \pi^{+} p$ at $2.75 \mathrm{GeV} / c$.

${ }^{13}$ Saclay-Orsay-Bari-Bologna Collaboration, Nuovo Cimento 35 713 (1965): $\pi^{-} p$ at $2.75 \mathrm{GeV} / c$.

${ }^{14}$ C. Alff, D. Berley, D. Colley, N. Gelfand, U. Nauenberg, D. Miller, J. Schultz, J. Steinberger, T. H. Tan, H. Brugger P. Kramer, and R. Plano, Phys. Rev. Letters 9, 322 (1962)

T. H. Tan, Columbia University Nevis Report No. 123, 1964 (unpublished): $\pi^{+} p$ at 2.35 and $2.90 \mathrm{GeV} / c$.

${ }_{15}$ Aachen-Berlin-Birmingham-Bonn-Hamburg-London-München Collaboration, Nuovo Cimento 34, 495 (1964): $\pi^{+} p$ at $4 \mathrm{GeV} / c$.

${ }_{16}$ Aachen-Berlin-Birmingham-Bonn-Hamburg-London-München Collaboration, Nuovo Cimento 31, 729 (1964): $\pi^{-} p$ at $4 \mathrm{GeV} / c$.

${ }_{17}$ Aachen-Berlin-CERN Collaboration, Phys. Letters 12, 356 (1964); and private communication: $\pi^{+} p$ at $8 \mathrm{GeV} / c$.

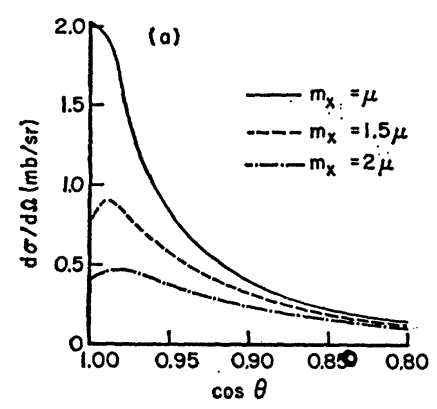

FIG. 1. (a) Differential cross section for the reaction $\pi^{+} p \rightarrow \rho^{+} p$ at 2.75 $\mathrm{GeV} / c$ as a function of $m_{x}$, the mass of the exchanged pseudoscalar particle. The absorption parameters are $C_{+}$ $=0.82, C_{-}=1.00, \quad \gamma_{+}$ $=0.062, \gamma_{-}=0.046$. The known coupling constants in the notation of Ref. 22 are $g^{2} \pi^{+} \pi^{0} \rho^{+}$ $4 \pi=2, G^{2} p \pi^{0} / 4 \pi=14.6$. (b) The $\rho$ meson's density-matrix $\rho_{00}$ for the same reaction.

depends appreciably on the mass $m_{x}$ of the exchanged particle once absorption is included. Figure 1(a) shows the differential cross section at $2.75 \mathrm{GeV} / c$ for $\rho$ production via pseudoscalar exchanges for $m_{x} / \mu=1.0,1.5,2.0$, while the density matrix element ${ }^{18} \rho_{00}$ is shown in Fig. $1(\mathrm{~b})$. Near the forward direction $d \sigma / d \Omega$ is exceedingly sensitive to $m_{x}$. The data are in agreement with the $m_{x}=\mu$ curve (see Fig. 4), and completely exclude a value of $m_{x}$ as large as $1.5 \mu$. The density matrix element $\rho_{00}$ shows a similar though considerably less pronounced dependence on $m_{x}$. The theoretical values of $\rho_{00}$ for $m_{x}=\mu$ are in good agreement with the data quoted in Sec. IIC below, whereas those for $m_{x}=2 \mu$ are not.

If one-particle exchange is the basic mechanism, the mass $m_{x}$ is not an adjustable parameter. The fact that agreement with experiment is only attained when $m_{x}$ is in the immediate vicinity of the pion mass gives one considerable confidence in the model.

From Fig. 1(a) we make the additional observation that the absorptive effects are unable to overcome the defocusing that results when $m_{x}$ is increased. This occurs when the ranges of the absorptive interactions are comparable to or larger than $1 / m_{x}$, because the diminution of the production amplitudes is then a slowly varying function of $j$. Thus in vector-mesonexchange processes, where $m_{x} \gg \mu$, we expect the absorptive effects to produce rather less collimation than in pion-exchange processes.

\section{B. Dependence on Absorption Parameters}

In all the reactions considered here, one (or more) of the reaction products is unstable. The parameters $C$

${ }^{18} \mathrm{~K}$. Gottfried and J. D. Jackson, Nuovo Cimento 33, 309 (1964). 


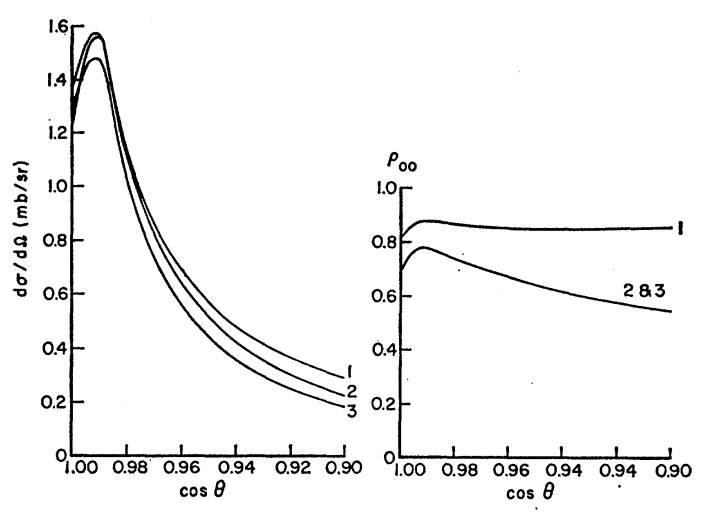

FIG. 2. Differential cross section and $\rho_{00}$ for the reaction $\pi^{-} p \rightarrow$ $\rho^{-} p$ at $4 \mathrm{GeV} / c$. The coupling constants are the same as in Fig. 1 . The absorption parameters for the initial state are $C_{+}=0.76$, $\gamma_{+}=0.040$ throughout. Those for the final state are, curve 1: $C_{-}=C_{+}, \gamma_{-}=\gamma_{+} ;$curve $2: C_{-}=1.00, \gamma_{-}=\gamma_{+} ;$curve $3: C_{-}=1.00$, $\gamma_{-}=0.75 \gamma_{+}$.

and $\gamma$ that characterize the elastic amplitudes are therefore only available for the initial state. Although photoproduction data will soon provide information concerning the final-state interactions, parameters $C_{-}$ and $\gamma_{-}$are at present unknown. We have therefore explored the sensitivity of $d \sigma / d \Omega$ and $\rho_{m m^{\prime}}$ to modest variations of $C_{-}$and $\gamma_{-}$for a number of different reactions. Typical effects are shown in Fig. 2 for $\pi^{-} p \rightarrow \rho^{-} p$ at $4 \mathrm{GeV} / c$. Three cases are considered: (1) identical initial- and final-state interactions; (2) total absorption for the final $S$ state together with equal ranges of the elastic absorptive interactions; (3) range of final-state interaction longer by a factor of $(0.75)^{-1 / 2}$ than that of initial state, together with total absorption in the $S$ wave.

From Fig. 2 we conclude that modest variations of the strength and range of the final-state interaction do not lead to significant changes in the spin-density matrix or the differential cross section. We shall mention the effects of considerably larger variations of $\gamma_{-}$when we deal with the reaction $\pi N \rightarrow \pi N^{*}$ below.

We must point out that according to the derivation of the reaction amplitude given in Sec. 2 of $\mathrm{I}$, it is not permissible to treat $C_{-}$and $\gamma_{\text {_ as }}$ independently variable parameters in reactions mediated by pion exchange. The factorized form (1) for the reaction amplitude is only valid when the ranges of the absorptive interactions satisfy $\sqrt{ } A_{ \pm} \gg m_{x}^{-1}$. This inequality is well satisfied in vector-meson exchange, but is badly violated in pion exchange. Nevertheless, we shall treat $C_{-}$and $\gamma_{-}$ as independent parameters throughout, since this provides us with the only simple way of incorporating final-state interactions which may be stronger and more long-ranged than initial-state interactions.

\section{Comparison with Experiment}

We shall now compare the model's predictions with the data on $\rho$ production. The reaction mechanism is
Fig. 3. Differential cross section for the reaction $\pi^{-} p \rightarrow$ $\rho^{-p}$ at $1.6 \mathrm{GeV} / c$. The absorptive parameters are $C_{+}$ $=0.90, \gamma_{+}=0.12$. A similar fit is obtained for the reaction $\pi^{-} p \rightarrow \rho^{0} n$, where isotopic-spin considerations show that the cross section is twice that for $\rho^{-}$production. The data are taken from Ref. 11.

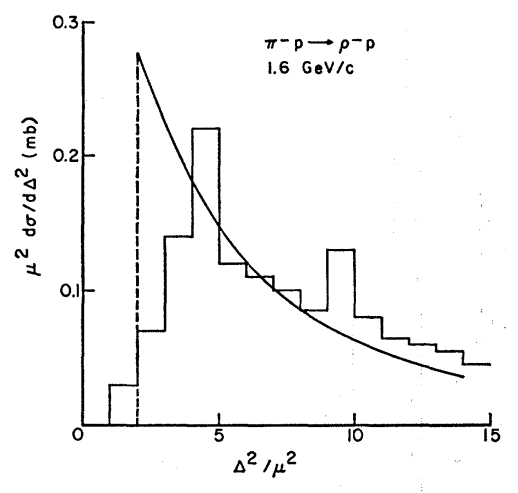

assumed to be purely pion-exchange; the coupling constants are then known (see I). In view of the fact that these predictions are not very sensitive to the precise values of $C_{-}$and $\gamma_{-}$, we have simply set $C_{-}=1$ and $\gamma_{-}=\frac{3}{4} \gamma_{+}$throughout, unless otherwise specified. This choice corresponds to a final-state interaction that is somewhat stronger and longer ranged than that in the initial state.

The comparison between theory and experiment for the differential cross section is shown in Figs. 3-6. The dependence of the total cross section on energy and charge is shown in Fig. 7. The difference between $\rho^{+}$ and $\rho-$ cross sections results from the different absorption parameters inferred from elastic $\pi p$ data. The peripheral model without absorption ${ }^{19}$ predicts equal cross sections for $\rho^{+}$and $\rho^{-}$production. Although the cross-section data are more or less consistent with both models, the $\rho^{+}$cross section appears to be systematically higher, in agreement with our calculations.

Data on the decay correlations for this reaction are relatively meager. At $2.75 \mathrm{GeV} / c$ the value of $\rho_{00}$, averaged over production angles, is $\left\langle\rho_{00}\right\rangle \simeq 0.7$ for both $\rho^{+}$and $\rho^{-}$production. ${ }^{12,13}$ A glance at Fig. 1(b) shows that our calculations are in excellent agreement with

Frg. 4. Differential cross section for the reaction $\pi^{+} p \rightarrow \rho^{+} p$ at 2.75 $\mathrm{GeV} / c$. The absorptive parameters are $C_{+}$ $=0.82, \gamma_{+}=0.062$. The data are taken from Ref. 12.

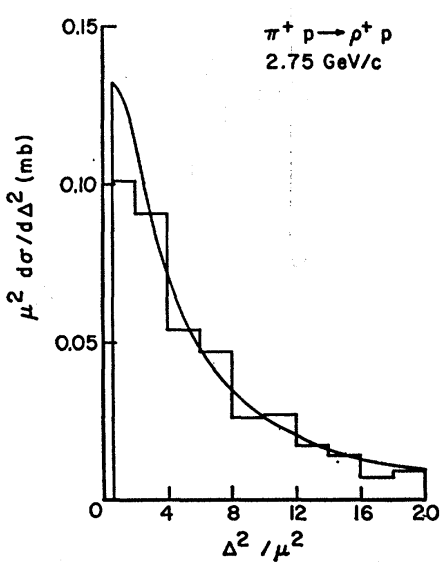

${ }^{19}$ U. Amaldi and F. Selleri, Nuovo Cimento 31, 360 (1964). 


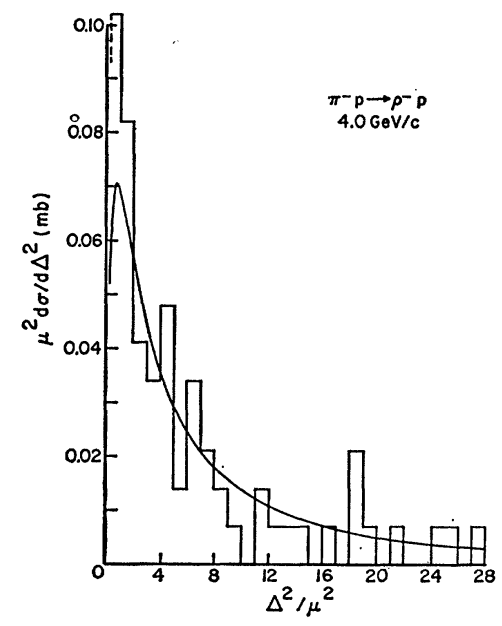

Fig. 5. Differential cross section for the reaction $\pi^{-} p \rightarrow \rho^{-} p$ at $4.0 \mathrm{GeV} / c$. The absorptive parameters are $C_{+}=0.76$ $\gamma_{+}=0.040$. The data are taken from Ref. 16.

these measurements. ${ }^{20}$ In $\rho^{+}$production at $4 \mathrm{GeV} / c$ the decay data $^{15}$ yields $\left\langle\rho_{00}\right\rangle=0.70 \pm 0.08,\left\langle\rho_{1,-1}\right\rangle=0.17$ $\pm 0.14,\left\langle\rho_{10}\right\rangle=-0.07 \pm 0.07$. The theoretical matrix elements averaged over $1 \leq \cos \theta \leq 0.7$ are $0.65,0.06$ and -0.18 , respectively. In $\rho^{-}$production at $4.0 \mathrm{GeV} / c$ the decay data ${ }^{21}$ averaged over the smaller angular interval $1 \leq \cos \theta \leq 0.9$ imply $\left\langle\rho_{00}\right\rangle=0.53 \pm 0.12,\left\langle\rho_{1,-1}\right\rangle=0.16$ $\pm 0.10,\left\langle\rho_{10}\right\rangle=-0.06 \pm 0.05$, and the corresponding theo-

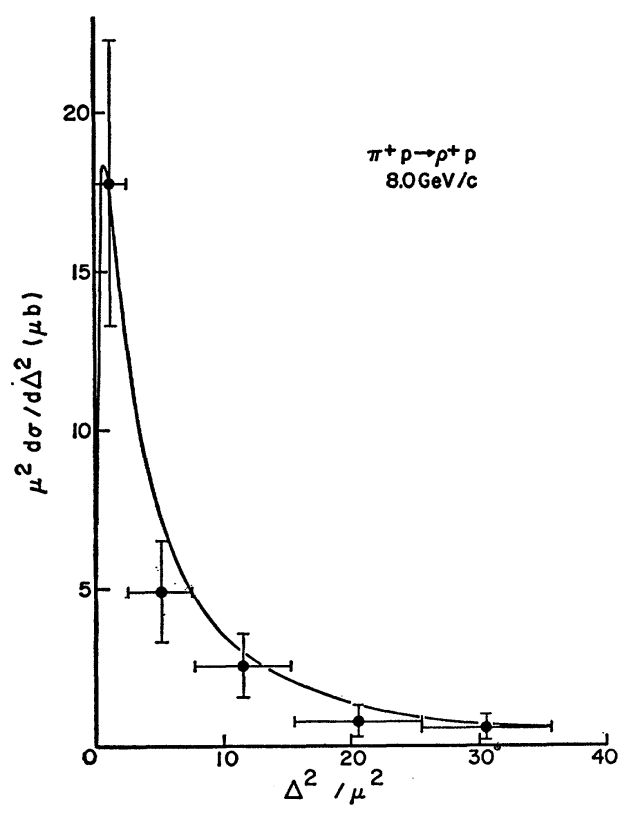

FIG. 6. Differential cross section for the reaction $\pi^{+} p \rightarrow \rho^{+} p$ at $8.0 \mathrm{GeV} / c$. The absorptive parameters are $C_{+}=0.60, \gamma_{+}=0.016$ The data are taken from Ref. 17.

${ }^{20}$ If $C_{-}$is kept equal to 1 , moderate variations of the other absorption parameters are barely visible in $\rho_{m m^{\prime}}$. We therefore do not show any curves for $\rho^{-}$production at $2.75 \mathrm{GeV} / c$. Furthermore, the values of $\rho_{m m^{\prime}}$ presented in I can be applied to both $\rho^{+}$ and $\rho^{-}$production at $4 \mathrm{GeV} / c$.

21 I. Derado, V. P. Kenney, and W. D. Shephard, Phys. Rev. Letters 13, 505 (1964). retical elements are $0.70,0.04$, and -0.17 , respectively.

The agreement between theory and experiment over the 1.6- to $8.0-\mathrm{GeV} / c$ range of incident momenta is, on the whole, highly satisfactory. It is true that the difference between the experimental total cross sections for $\pi^{+} p \rightarrow \rho^{+} p$ and $\pi^{-} p \rightarrow \rho^{-} p$ is considerably larger than that of the theory. It must, however, be remembered that this type of comparison involves absolutecross-section data from different experimental groups and also depends on ill-defined $\rho$-production cross sections at large momentum transfers, differences in background subtractions, etc. It is slightly disconcerting that the theory does not reproduce the forward minimum observed at $1.6 \mathrm{GeV} / c$. But this is probably

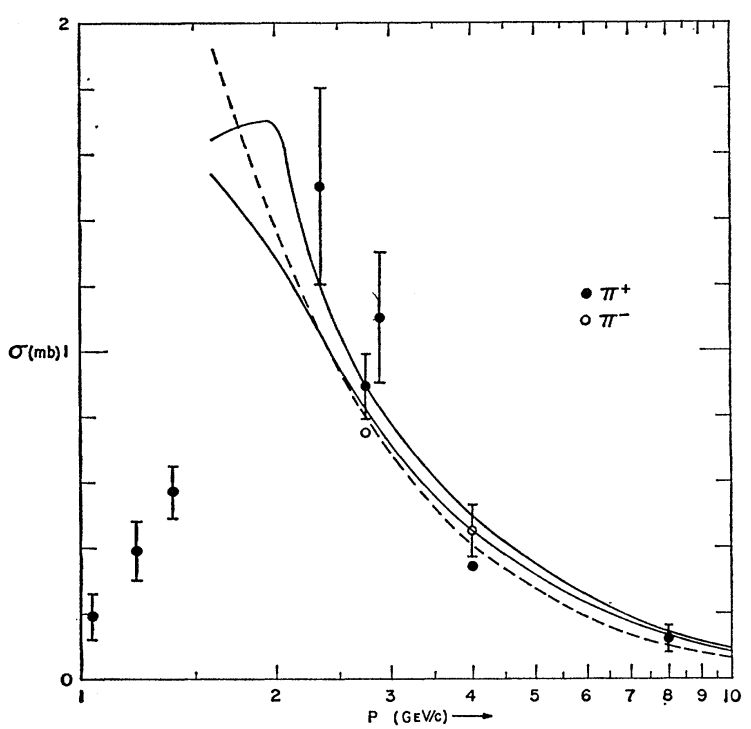

Fig. 7. The total cross section for the reaction $\pi^{ \pm} p \rightarrow \rho^{ \pm} p$ as a function of incident pion momentum, assuming only pion exchange. The upper (lower) solid curve is for $\pi^{+}\left(\pi^{-}\right)$, the difference being caused by different absorptive effects. The dashed curve is the cross section of the one-pion-exchange model with an empirical form factor [see Eq. (41) of Ref. 19]. The experimental points are taken from Refs. 10, 12-17.

too low an energy for the peripheral model, and even more so for the high-energy approximations used by us.

In I we predicted that the $\rho$ 's density matrix should deviate significantly from the one given by the conventional peripheral model (i.e., $\rho_{00}=1$, all other elements zero). The decay data just quoted confirm this.

\section{Vector Exchange}

The foregoing discussion has, in its entirety, been based on the assumption that only pion exchange is involved. There is no theoretical foundation for this assumption: It is based on the early experimental observation ${ }^{16}$ that the $\rho$ meson produced in this reaction displays a rather flat Treiman-Yang distribution, and the fact that, apart from a factor of roughly 2 in pro- 
duction rate, the reactions $\pi^{-} p \rightarrow \rho^{-} p$ and $\pi^{-} p \rightarrow \rho^{0} n$ are indistinguishable. ${ }^{16}$ But vector-meson exchange appears to be a rather common phenomenon in peripheral reactions. We have therefore investigated the sensitivity of our results to various admixtures of vector exchange. There are two coupling constant combinations which determine the strength of vector exchange relative to the pseudoscalar exchange:

$$
\begin{gathered}
\xi=\frac{f_{\pi \rho V}\left(G_{V_{\bar{p} p}}+G^{T} V_{\bar{p} p}\right)}{2 g_{\pi \pi \rho} G_{\pi \bar{p} p}}, \\
\eta=f_{\pi \rho} G^{T} V_{\bar{p} p} / g_{\pi \pi \rho} G_{\pi \bar{p} p},
\end{gathered}
$$

where the superscript designates vector or tensor coupling. ${ }^{22}$ Because of $G$-parity invariance, only $\varphi$ and $\omega$ exchange can enter into the reaction $\pi N \rightarrow \rho N$. By analogy with the isoscalar electromagnetic form factors, we take $G^{T} \ll G^{V}$, or $\eta \simeq 0 .^{23}$ The results are shown for $\xi= \pm 0.25$ and $\xi= \pm 0.50$ in Fig. 8 assuming $m_{V}=m_{\omega}$. The presence of vector-exchange causes the differential cross section to increase and the density matrix element $\rho_{00}$ to decrease at angles away from the forward direction. Another effect, not shown in Fig. 8, is a sizeable azimuthal decay parameter $\rho_{1,-1}$ (e.g., with $\xi= \pm 0.50$ at $4 \mathrm{GeV} / c$ or $\xi= \pm 0.25$ at $\left.8 \mathrm{GeV} / c,\left\langle\rho_{1,-1}\right\rangle \simeq 0.3\right)$. The importance of vector exchange increases dramatically with energy: whereas $|\xi|=0.25$ gives a barely acceptable fit at $4 \mathrm{GeV} / c$, it is far too large at $8 \mathrm{GeV} / c^{24}$

${ }^{22}$ The definitions of these coupling constants are the same as in J. D. Jackson and H. Pilkuhn, Nuovo Cimento 33, 906 (1964); 34, 1841E (1964).

${ }^{23}$ This choice depends on the questionable assumption that the mixture of $\omega$ and $\varphi$ that enters here is the same as in the electromagnetic interaction. That $\omega$ exchange dominates in both processes is suggested by the large contribution of the $\omega$ pole to the isoscalar form factor, and by the abnormally small branching ratio for $\varphi \underset{24}{\rightarrow} \pi \rho$.

ing pure $\omega$ exchange, and accepting the estimate $f^{2}{ }_{\omega \rho \pi} / 4 \pi \simeq 10$ based on the idea the $\omega \rightarrow \rho \pi$ dominates the $\omega \rightarrow 3 \pi$ decay [M. Gell-Mann, D. Sharp, and W. G. Wagner, Phys. Rev. Letters 8, $261(1962)]$, we find that $|\xi|=0.25$ corresponds to $\left(G^{V}{ }_{\omega N N}\right)^{2} / 4 \pi \simeq 0.7$. This can be compared with estimates derived from nuclear force calculations, $\left(G^{V}{ }_{\omega N N}\right)^{2} / 4 \pi \simeq 3$ [A. Scotti and D. Y. Wong, Phys. Rev. Letters 10, 142 (1963); Phys. Rev. 138, B145 (1963)], $\left(G^{V} \omega N N\right)^{2} / 4 \pi \simeq 22$ [R. A. Bryan and B. L. Scott, Phys. Rev. 135, B434 (1964) ]. In the light of this comparison one might well ask whether $|\xi|$ can be determined in other ways. This cannot be done within the framework of $S U_{3}$ because of the existence of vector mesons belonging to both the octet and singlet representations. $S U_{6}$ and its relativistic generalizations $[\mathrm{F}$. Gürsey and L. A. Radicati, Phys. Rev. Letters 13, 173 (1964); F. Gürsey, A. Pais, and L. A. Radicati, ibid. 13, 299 (1964); B. Sakita, ibid. 13, 643 (1964); Phys. Rev. 136, B1756 (1964); A. Salam, R. Delbourgo, and J. Strathdee, Proc. Roy. Soc. (London) A284, 146 (1965); M. A. B. Bég and A. Pais, Phys. Rev. Letters 14, 267, 509 (1965); B. Sakita and K. C. Wali, ibid. 14, 404 (1965); K. Bardakci, J. M. Cornwall, P. G. O. Freund, and B. W. Lee, ibid. 14, 48 (1965); ibid., p. 264 (1965)] relate the singlet and octet representations and yield a vanishing $\rho \pi \varphi$ coupling. Furthermore, the Dirac and Pauli couplings of $\rho^{0}$ and $\omega$ to $\tilde{p} p$ are identical, while the $\varphi \tilde{p} p$ couplings are such that the octet isoscalar vector meson $\left(\omega_{8}\right)$ has a small Pauli coupling $\left(G^{T} \omega_{8} \bar{p} p \simeq 0\right)$, consistent with the isoscalar anomalous magnetic moment. From this viewpoint the curves of Fig. 8 should be taken only as qualitative indications of the effects of vector exchange; within $S U_{6}$ only $\omega$ exchange can occur, with $\eta / \xi \simeq 2 \times 3.71 / 4.71=1.575$.
The significance of estimates of $|\xi|$ deduced from the highest energy data is, however, questionable in view of the difficulties with the energy dependence of vectormeson exchange discussed above.

\section{III. $K N \rightarrow K^{*} N$ AND $\bar{K} N \rightarrow \bar{K}^{*} N$}

The CERN experiments ${ }^{25}$ on the decay of $K^{*}$ produced in the reaction $K p \rightarrow K^{*} p$ at $3.0 \mathrm{GeV} / c$, when analyzed on the basis of the peripheral model without absorption, ${ }^{22}$ imply that the reaction mechanism is strongly dominated by vector-meson exchange. The earlier ${ }^{26} 1.96-\mathrm{GeV} / c$ data on this reaction, when analyzed in the same manner, do reveal a noticeable admixture of pseudoscalar exchange. A considerable amount of new and very interesting data is now becoming available on $K p \rightarrow K^{*} p$ and closely related reactions. We refer, in particular, to the very detailed CERN analysis ${ }^{27}$ of decay correlations in $K p \rightarrow K^{*} p$ at 3.0 $\mathrm{GeV} / c$, the preliminary CERN data ${ }^{28}$ on $K p \rightarrow K^{*} p$ at $5.0 \mathrm{GeV} / c$, the Berkeley deuterium experiment ${ }^{29}$ which yields information on $K^{+} n \rightarrow K^{*} p$ at $2.3 \mathrm{GeV} / c$, and the Saclay data ${ }^{30}$ on $K^{-} p \rightarrow \bar{K}^{*} N$ at $3.0 \mathrm{GeV} / c$. We shall have something to say about all of these data presently, but for the moment we confine the discussion to $K p \rightarrow K^{*} p$ at $3 \mathrm{GeV} / c$.

Just as for $\pi N \rightarrow \rho N$, the strength of the pion-exchange interaction is known in the reaction $K N \rightarrow K^{*} N$. As far as the vector-coupling constants are concerned, the ambiguities associated with $\omega-\varphi$ mixing (when $T=0$ exchange is allowed) are compounded by the possibility of $\rho$ exchange. There is therefore no $a$ priori information concerning the coupling parameters $\xi$ and $\eta$ defined by expressions analogous to (7) and (8). For definiteness we have taken the vector-meson mass to be $750 \mathrm{MeV}$. Changes of this parameter can be compensated by small alterations of the coupling constants.

\section{A. $K^{+} p \rightarrow K^{*} p$ at $3.0 \mathrm{GeV} / c$}

We have made a systematic search for those domains in the $\xi-\eta$ plane where a satisfactory fit to the production angular distribution is obtained for $K^{+} p \rightarrow K^{*} p$

${ }^{25}$ G. R. Lynch, M. Ferro-Luzzi, R. George, Y. GoldschmidtClermont, V. P. Henri, B. Jongejans, D. W. G. Leith, F. Muller, and J.-M. Perreau, Phys. Letters 9, 359 (1964).

${ }^{26}$ S. Goldhaber, Proceeding of the Athens Topical Conference on Recently Discovered Resonant Particles, edited by B. A. Munir and L. J. Gallaher (Ohio University Press, Athens, Ohio, 1963), p. 92.

${ }_{27}$ M. Ferro-Luzzi, R. George, Y. Goldschmidt-Clermont, V. P. Henri, B. Jongejans, D. W. G. Leith, G. R. Lynch, F. Muller, and J.-M. Perreau, Nuovo Cimento 36, 1101 (1965).

28 Private communication from the CERN group of Refs. 25 and 27.

${ }^{29}$ S. Goldhaber, I. Butterworth, G. Goldhaber, A. A. Hirata, J. A. Kadyk, T. A. O'Halloran, B. C. Shen, and G. H. Trilling, in Proceedings of the 12th Annual International Conference on HighEnergy Physics, Dubna, 1964 (Moscow, 1965).

${ }^{30}$ R. Barloutaud, A. Leveque, C. Louedec, J. Meyer, P. Schlein, A. Verglas, J. Badier, M. Demoulin, J. Goldberg, B. P. Gregory, P. Krejbich, C. Pelletier, M. Ville, E. S. Gelsema, J. Hoogland, J. C. Kluyver, and A. G. Tenner, Phys. Letters 12, 352 (1964). 
B 434 J ACKSON, DONOHUE, GOTTFRIED, KEYSER, A N S E E N S ON

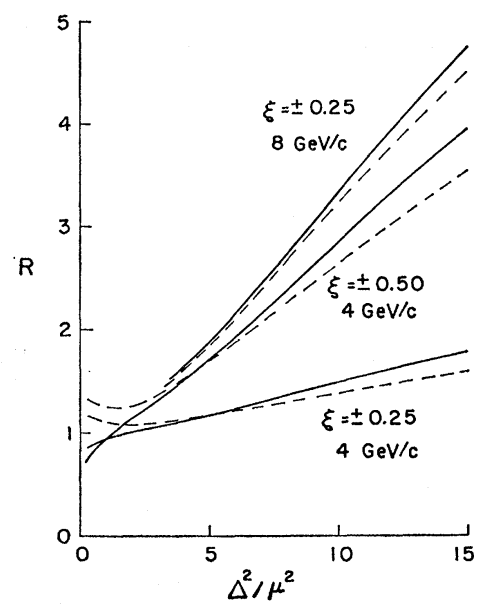

at $3 \mathrm{GeV} / c^{31}$ As is shown in Fig. 9, there are two very distinct domains where $\chi^{2}$ is acceptable. Theoretical production angular distributions typical of the two

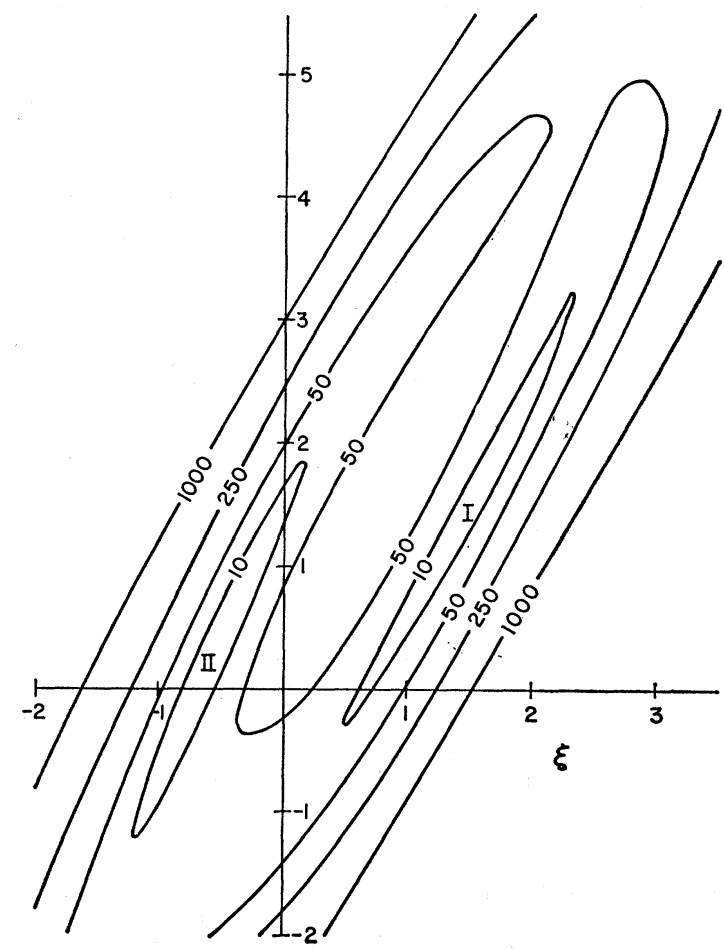

FIG. 9. Contour diagram of $\chi^{2}$ in the $(\xi, \eta)$ plane for a fit to the differential cross section of the reaction $K^{+} p \rightarrow K^{*} p$ at $3 \mathrm{GeV} / c$. Seven data points from Ref. 25, covering $1 \leq \cos \theta \leq 0.65$, are used. The absorption parameters are $C_{+}=0.90, \gamma_{+}=0.11$. The known pion-exchange coupling constants are $g^{2} K^{+} \pi^{0} K^{*} / 4 \pi=0.75$, $G_{p p \pi^{0}}^{2} / 4 \pi=14.6 .|\xi|=1$ corresponds to

and

$$
\left(f_{K^{+} V^{0} K^{*}}\left(G^{V}{ }_{p p V^{0}}+G^{T} p p V^{0}\right) / 4 \pi\right)^{2}=44 \text {, }
$$

$$
|\eta|=1 \text { to }\left(f G^{T} / 4 \pi\right)^{2}=11 \text {. }
$$

Only two regions of small $\chi^{2}$, labeled I and II, are found. Along the valley in domain I(II) the smallest $\chi^{2}$ is 3.5 (4.7), compared with an expected value of 5 .

${ }^{31}$ In carrying out this search we keep $C_{-}=1.00$ and $\gamma_{-}=0.75 \gamma_{\gamma_{+}}$. Moderate alterations of these absorption parameters can be compensated by a slight shift of the domains shown in Fig. 9, and would not alter our conclusion to a significant degree.
Fig. 8. The dependence of the differential cross section and $\rho_{00}$ on the strength of vector-meson exchange in the reaction $\pi p \rightarrow \rho p$ at 4 and 8 $\mathrm{GeV} / c$. All curves are for $\eta=0$. The absorption parameters are the same as in Figs. 5 and 6 . The solid (dashed) curves are for $\xi$ positive (negative), corresponding to destructive (constructive) interference in the forward direction. $R$ is the ratio of the differential cross section with pion and vectormeson exchange to that for pion exchange alone $(\xi=0)$. The $\rho_{00}$ curves for $\xi= \pm 0.25$ at $8 \mathrm{GeV} / c$ are not shown; they lie closely on either side of the lowest dashed curve. domains are compared with the $3-\mathrm{GeV} / c$ data in Fig. 10. The decay correlations can be accounted for with the same coupling parameters as give satisfactory fits to the differential cross section. The density matrix is found to be a slowly varying function of $\xi$ and $\eta$; curves characteristic of the two domains of the $\xi-\eta$ plane mentioned above are compared with experiment ${ }^{27}$ in Fig. 11. Within the large statistical errors, the accord between theory and the data is satisfactory. ${ }^{32}$

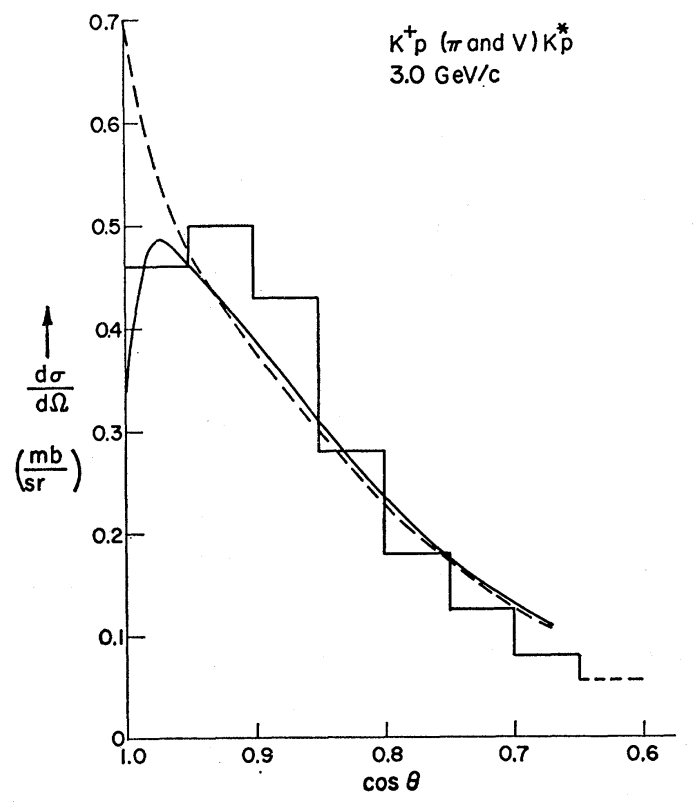

FIG. 10. Comparison of the theoretical differential cross section of $K^{+} p \rightarrow K^{*} p$ (with $K^{*} \rightarrow K^{0} \pi^{+}$) at $3 \mathrm{GeV} / c$ with the experimental data (Ref. 25). The absorption parameters are the same as in Fig. 9. The solid (dashed) curve corresponds to vectormeson coupling constants in domain I (II) of Fig. 9. The actual values are $\xi=1.55, \eta=1.48(\xi=-0.72, \eta=0.10)$, but the curves change very slowly as $\xi$ and $\eta$ are moved along the long and narrow valleys of domains I and II.

${ }^{32}$ In the peripheral model without absorption, there is no interference between pseudoscalar and vector exchange (Ref. 22) in either the differential cross section or the decay correlations. This is no longer true when absorption is included. For further details see Fig. 16 of Ref. 7 and accompanying discussion. 

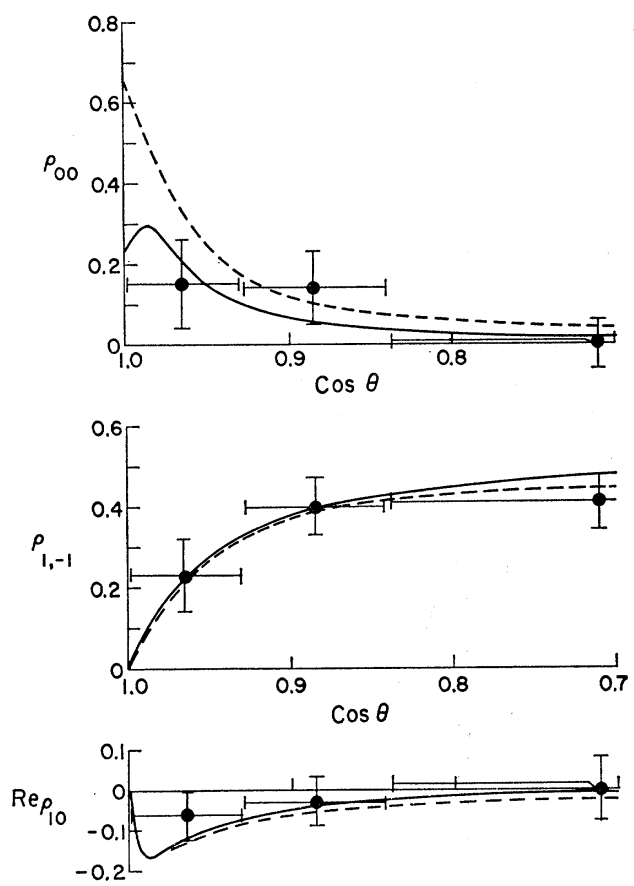

FIG. 11. Theoretical and experimental (Ref. 27) $K^{*}$ densitymatrix elements for the reaction of Fig. 10 as a function of centerof-mass production angle. The solid and dashed curves correspond to those of Fig. 10.

\section{B. Isospin Character of Vector Exchange}

Both isoscalar and isovector exchange are possible in $K^{+} p \rightarrow K^{*} p$, but only the latter is allowed in the reaction $K^{+} n \rightarrow K^{*} p$. The Berkeley $K^{+}-d$ experiment ${ }^{29}$ comparing these two reactions therefore yields information on the isotopic spin character of the exchange process. The decay correlations in $K^{+} n \rightarrow K^{*} p$ are characteristic of pseudoscalar exchange, whereas in $K^{+} p \rightarrow K^{*} p$ they are typical of appreciable vector exchange as expected from the $3-\mathrm{GeV} / c$ experiment on this reaction. In the light of this experimental result, one may ask whether a choice can be made between the two $\xi-\eta$ domains of Fig. 9 that fit the charge-zero transfer process. The only firm statement that can be made (based on the interference from isovector electromagnetic form factors that $G^{T} \gg G^{V}$ for $\rho$ exchange) is that domain II of Fig. 9 cannot correspond to pure $\rho$ exchange. Because the ratio $G^{T} / G^{V}$ is unknown for isoscalar exchange, a preference of one $\xi-\eta$ domain over the other cannot be established without further assumptions. ${ }^{33}$

${ }^{33}$ (a) $S U_{3}$ does not help because it does not relate the octet and singlet vector meson couplings, while the electromagnetic data only provide $G^{T} / G^{V}$ for the octet. (b) $S U_{6}$ does make specific predictions, as discussed in footnote 24 . The relativistic theory allows the exchange of $\rho, \omega$ and $\varphi$ mesons (for $Q_{\mathrm{ex}}=0$ ) with coupling constants such that the coherent superposition of the three amplitudes for $K^{+} p \rightarrow K^{*} p$ is equivalent to the exchange of a single vector meson with $G^{V}=0$, i.e., $\eta=2 \xi$. The line $\eta=2 \xi$ lies squarely along the ridge of large $\chi^{2}$ between domains I and II in Fig. 9. For processes with $Q_{\mathrm{ex}}=1$ (e.g., $K^{+} n \rightarrow K^{* 0} p$ ) the $\rho$-ex-
A comparison of the theory with the $2.3-\mathrm{GeV} / c$ Berkeley data on cross sections and decay angular distributions is shown in Fig. 12. For $K^{+} n \rightarrow K^{*} p$ only pion exchange is assumed, whereas for $K^{+} p \rightarrow K^{*} p$ vector exchange is included with the coupling constants of domains I and II of Fig. 9. The $K^{+} n \rightarrow K^{*} p$ results are fitted well by pion exchange; the $K^{+} p \rightarrow K^{*} p$ data favor the vector-coupling constants of domain I.

\section{C. $K^{-} p \rightarrow \bar{K}^{*} p$ at $3 \mathrm{GeV} / c$}

If our model is correct the coupling constants found in the analysis of $K^{+} p \rightarrow K^{*} p$ should be appropriate to $\bar{K}^{*}$ production in $K^{-} p$ collisions. The experimental results on $K^{*}$ and $\bar{K}^{*}$ production at $3 \mathrm{GeV} / c$ are virtually indistinguishable. ${ }^{30}$ From our viewpoint differences between $K^{+} p \rightarrow K^{*} p$ and $K^{-} p \rightarrow \bar{K}^{*} p$ should arise because the $K^{-}-p$ elastic cross section is much more sharply peaked and the total cross section considerably larger than those for $K^{+}-p$. The absorptive parameters inferred from $K^{+}-p$ and $K^{-}-p$ elastic scattering at $3 \mathrm{GeV} / c^{34,35}$ are

$$
\begin{array}{ccc} 
& C_{+} & \gamma_{+} \\
K^{+} p: & 0.90 & 0.110 \\
K^{-} p: & 0.64 & 0.050
\end{array}
$$

As expected, the absorptive interaction for $K^{-}-p$ extends to larger impact parameters than for $K^{+}-p$ but, surprisingly enough, is far from totally absorbing in the lowest partial waves.

Searches in the $\xi-\eta$ plane were made for satisfactory fits to the experimental differential cross section at $3 \mathrm{GeV} / c^{30}$ with our conventional choice $C_{-}=1$ and $\gamma_{-}=\frac{3}{4} \gamma_{+}$, as well as for smaller values of $C_{-}$. Because $C_{+}$is small, the theoretical angular distribution is rather sensitive to $C_{-}$; total absorption in the final-state $S$ wave appears to be required for an acceptable fit. Even when $C_{-}=1$ the best fits are not very good. With the amount of vector exchange necessary to fit the absolute cross section in the forward direction, the angular distribution is too broad. If advantage is taken of the $20 \%$ error quoted on the reaction cross section $(0.75 \pm 0.15$ $\mathrm{mb}$ ) and a search is made for fits to the data multiplied by 0.8 , quite acceptable solutions are found in the general locations of domains I and II of Fig. 9, but with somewhat larger absolute values of $\xi$ and $\eta$. A

change contribution is predicted to have a $\xi$ value $5 / 12$ ths as large as the equivalent $\xi$ for $Q_{\mathrm{ex}}=0$. A vector-exchange contribution of this magnitude may be just consistent with the data shown in Fig. 12. (c) The notion of amplitude parity [J. B. Bronzan and F. E. Low, Phys. Rev. Letters 12, 522 (1964)] is of no assistance. It ascribes the isoscalar electromagnetic form factor to $\varphi$ exchange, but implies that the dominant vector meson exchanged in the reaction considered in Secs. II and III is the $\omega$.

${ }^{34}$ J. Debaisieux, F. Grard, J. Heughebaert, L. Pape, R. Windmolders, M. Ferro-Luzzi, R. George, Y. Goldschmidt-Clermont, V. P. Henri, B. Jongejans, D. W. G. Leith, G. R. Lynch, F. Muller, J.-M. Perreau, G. Otter, and P. Sällström, in Proceedings of the 12th Annual Conference on High-Energy Physics, Dubna, 1964 (Moscow, 1965).

${ }_{35}$ M. N. Focacci, S. Focardi, G. Giacomelli, L. Monari, and P. Serra (unpublished). 

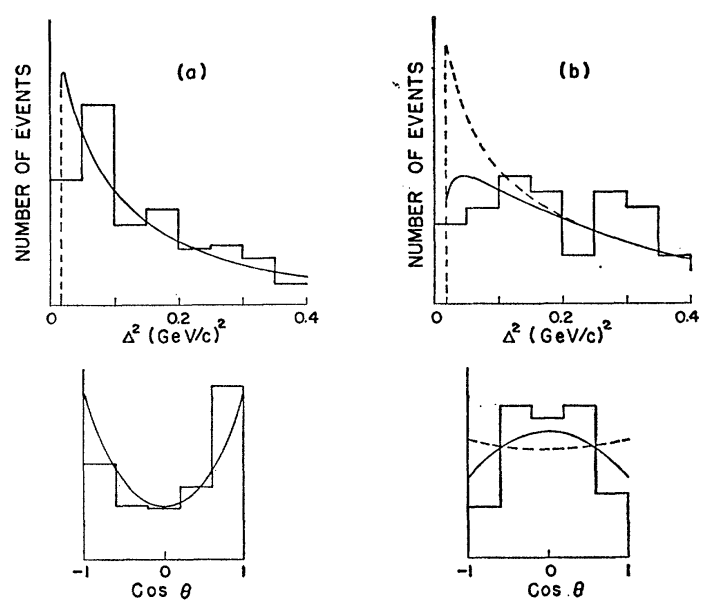

FIG. 12. Differential cross sections (above) and $K^{*}$ decay angular distributions (below) for the reactions $K^{+} n \rightarrow K^{* 0} p$ and $K^{+} p \rightarrow K^{*+} p$ at $2.3 \mathrm{GeV} / c$. The data are from Ref. 29. Part (a) is for $K^{+} n \rightarrow K^{* 0} p$, with the curves calculated assuming only pion exchange. Part (b) is for $K^{+} p \rightarrow K^{*+} p$, with curves computed for pion and vector-meson exchange with the latter's coupling constants as found at $3 \mathrm{GeV} / c$ [solid (dashed) curve for domain I (II) of Fig. 9]. The abosrption parameters are $C_{+}=1.0, \gamma_{+}=0.17$.

compromise choice of $\xi$ and $\eta$ can be made that gives reasonable agreement with both the $K^{+}$and $K^{-}$data, within the limits of error on the absolute normalizations. Figure 13 shows the differences that arise because of different absorptive effects. The coupling constants

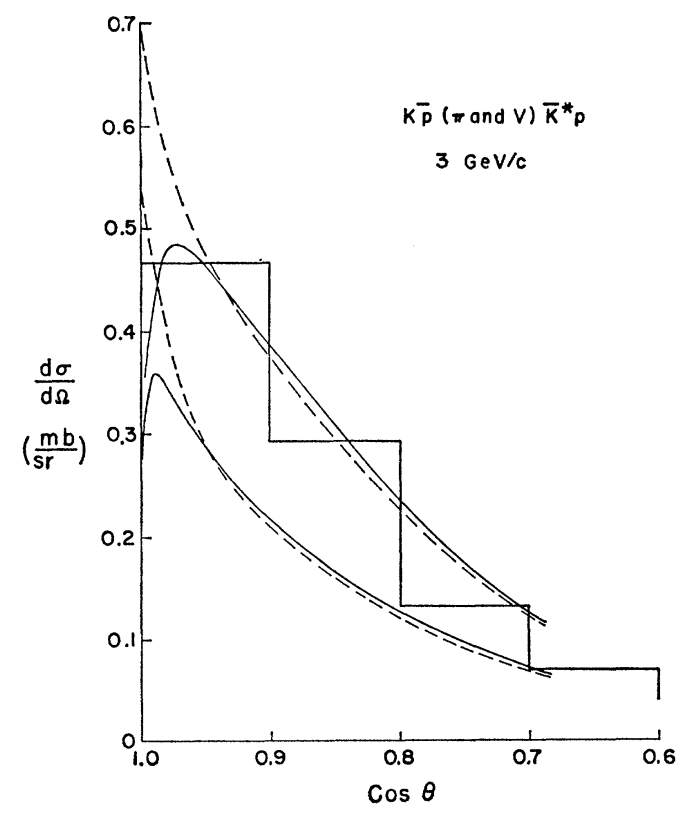

FIg. 13. Theoretical and experimental (Ref. 30) differential cross sections for the reaction $K^{-} p \rightarrow \bar{K}^{*-} p$ (with $\bar{K}^{*-} \rightarrow \bar{K}^{0} \pi^{-}$) at $3 \mathrm{GeV} / c$. The upper set of solid and dashed curves are those of Fig. 10 for $K^{+} p \rightarrow K^{*+} p$. The lower pair are calculated with the same coupling constants as in Fig. 10 , but with $K^{-} p$ absorption parameters, $C_{+}=0.65, \gamma_{+}=0.050, C_{-}=1.0, \gamma_{-}=0.038$. are those found for the $K^{+} p$ data at $3 \mathrm{GeV} / c$. The angular variation of the $\bar{K}^{*}$ density matrix is compared with the data ${ }^{30}$ in Fig. 14. On the whole the agreement is satisfactory within statistical uncertainties.

\section{D. $K^{+} p \rightarrow K^{*} p$ at $5 \mathrm{GeV} / c$}

Until now we have discussed the reaction $K p \rightarrow K^{*} p$ at $3 \mathrm{GeV} / c$ and at $2.3 \mathrm{GeV} / c$. There are data available at ${ }^{26} 1.96 \mathrm{GeV} / c$ and preliminary results at $5 \mathrm{GeV} / c$ from the CERN group. ${ }^{28} \mathrm{~A}$ comparison between the $5-\mathrm{GeV} / c$ data and our theoretical cross section is shown in Fig. 15. The coupling constants are those of Figs. 9
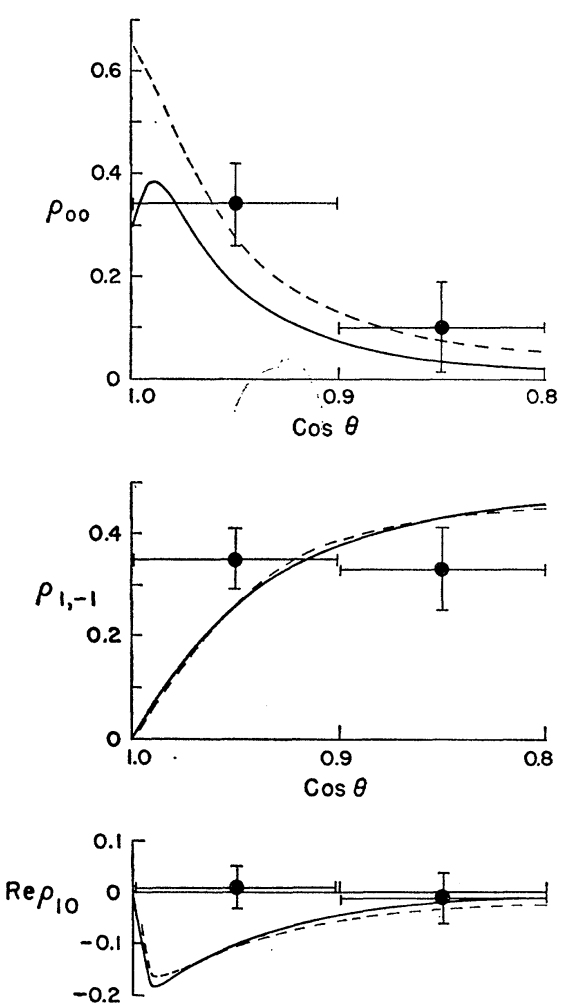

FIG. 14. Comparison of theoretical and experimental (Ref. 30) $\bar{K}^{*}$ density-matrix elements as a function of production angle for the reaction of Fig. 13. The solid (dashed) curve corresponds to vector-meson coupling constants from domain I (II) of Fig. 9, but with the $K^{-} p$ absorption parameters given in Fig. 13.

and 10 , determined at $3 \mathrm{GeV} / c$. Although the agreement is satisfactory for small momentum transfers $\left(\Delta^{2} \leqslant 15 \mu^{2}\right)$, the theoretical cross section is much too high at larger values of $\Delta^{2}$. This is reflected in the theoretical total cross section of $0.85 \mathrm{mb}$, compared with the experimental estimate of approximately $0.3 \mathrm{mb}$. One can argue that the model, and the approximations used by us, are only expected to be valid at small $\Delta^{2}$. But at $3 \mathrm{GeV} / c$ we did achieve an excellent fit to the data over the interval, $0 \leq \Delta^{2} \leqslant 30 \mu^{2}$. The failure at $5 \mathrm{GeV} / c$ can, in all probability, be traced to the growth of the vectorexchange amplitude with energy. The disagreement 
should become more pronounced at higher energies.

The $K^{*}$ decay correlations have also been analyzed at $5 \mathrm{GeV} / c{ }^{28}$ The preliminary experimental results are $\left\langle\rho_{00}\right\rangle=0.25 \pm 0.12,\left\langle\rho_{1,-1}\right\rangle=0.42 \pm 0.11, \operatorname{Re}\left\langle\rho_{10}\right\rangle=-0.04$ \pm 0.09 . The corresponding theoretical values, averaged over the interval, $0 \leq \Delta^{2} \leq 20 \mu^{2}$, for the two domains of Fig. 9 are $\left\langle\rho_{00}\right\rangle=0.01,\left\langle\rho_{1,-1}\right\rangle=0.41, \operatorname{Re}\left\langle\rho_{10}\right\rangle=-0.02$ (domain I), and $\left\langle\rho_{00}\right\rangle=0.08,\left\langle\rho_{1,-1}\right\rangle=0.42, \operatorname{Re}\left\langle\rho_{10}\right\rangle$ $=-0.03$ (domain II). The only disagreement is for the value of $\rho_{00}$, and even there the comparison is sensitive to the averaging interval. ${ }^{36}$

On the whole the model achieves quite satisfactory agreement with a rather large body of experimental information currently available on the reactions $K^{+} N \rightarrow K^{*} N$ and $K^{-} p \rightarrow \bar{K}^{*} p$. Minor discrepancies occur, but they cannot be regarded as serious until substantiated by much greater statistical accuracy, especially for the decay correlations. The most glaring

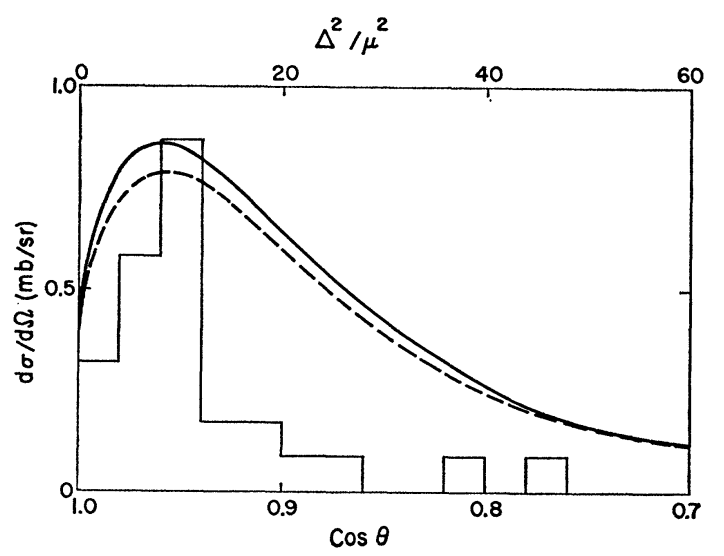

FIG. 15. Differential cross section for the reaction $K^{+} p \rightarrow K^{*} p$ at $5 \mathrm{GeV} / c$. The preliminary data are from Ref.28. The curves are calculated with the same coupling constants as at $3 \mathrm{GeV} / c$ (those of Fig. 10). The absorption parameters are $C_{+}=0.74, \gamma_{+}=0.050$.

failure is the disagreement with the angular distribution of production and magnitude of the reaction cross section at $5 \mathrm{GeV} / c$.

\section{ISOBAR PRODUCTION}

\section{A. $K^{+} p \rightarrow K^{0} N^{*}$ at $3 \mathrm{GeV} / c$}

We now turn to the CERN data ${ }^{27}$ on $N^{*}$ production in $K^{+} p$ collisions at $3.0 \mathrm{GeV} / c$. This reaction can only be mediated by the exchange of states with nonzero spin and isospin, i.e., via $\rho$ exchange in the simple model. There are then three independent coupling constants at the baryon vertex. We shall, however, restrict ourselves to the relativistic version ${ }^{22}$ of the magnetic-dipole model

${ }^{36}$ While for domain I of Fig. 9, the maximum value of $\rho_{00}$ is of the order of 0.06 , for domain II $\rho_{00}$ rises to a maximum of 0.52 in the forward direction. For this solution, averaging over the smaller interval $0<\Delta^{2}<10 \mu^{2}$ yields a value $\left\langle\rho_{00}\right\rangle=0.14$.

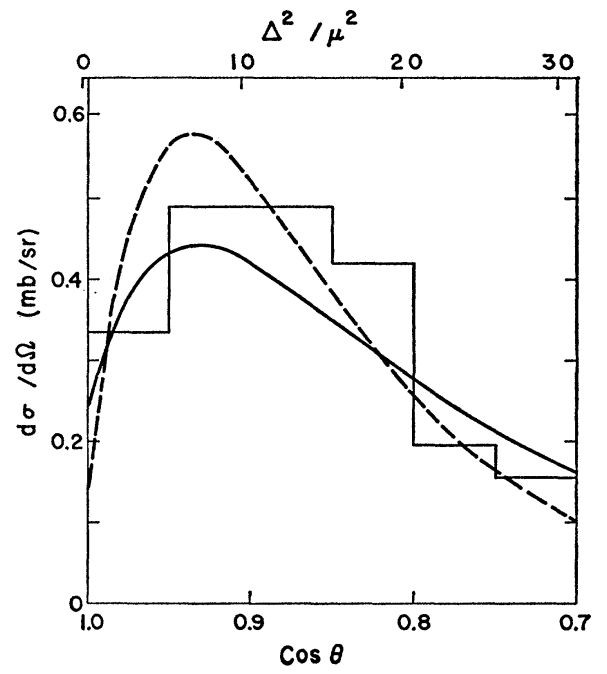

FIG. 16. Differential cross section for the reaction $K^{+} p \rightarrow K^{0} N^{*}$ at $3 \mathrm{GeV} / c$. The data are from Ref. 27. The solid curve is calculated using the relativistic version (Ref. 22) of the StodolskySakurai magnetic dipole model of $\rho$ exchange with the absorption parameters given in Fig. 10. The dashed curve shows the effect of a relatively modest intrinsic form factor, $F\left(\Delta^{2}\right)=M^{2} /\left(\Delta^{2}+M^{2}\right)$ with $M=0.9 \mathrm{GeV}$.

proposed by Stodolsky and Sakurai ${ }^{37}$ in which there is only one coupling constant.

The theoretical differential cross section is in good agreement with the data ${ }^{27}$ (see the solid curve in Fig. 16). The $N^{*}$ density matrix predicted by the model is compared to the data in Fig. 17. The agreement is

FIG. 17. Elements of the $N^{*}$ density matrix for the reaction $K^{+} p \rightarrow K^{0} N^{*}$ at $3 \mathrm{GeV} / c$ as functions of production angle. The data are from Ref. 27. The solid and dashed curves have the same meaning as in Fig. 16. The dotted curve in the top figure is the prediction of the relativistic magnetic dipole model of $\rho$ exchange without absorption (Ref. 22).
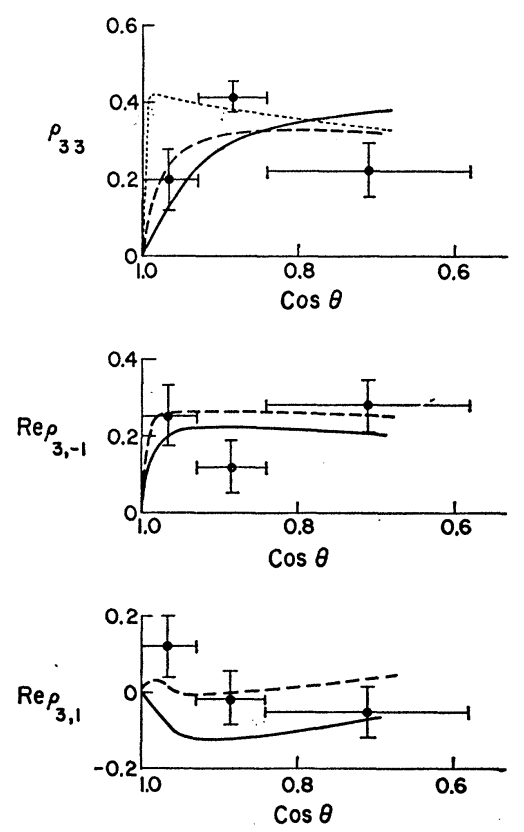

${ }^{37}$ L. Stodolsky and J. J. Sakurai, Phys. Rev. Letters 11, 90 (1963); L. Stodolsky, Phys. Rev. 134, B1099 (1964). 
qualitative at best. But it is obvious that more precise data are necessary for a decisive comparison between theory and experiment. ${ }^{38}$

As discussed in Sec. 3 of Ref. 22, the $\rho$-photon analogy and $S U_{3}$ lead to the coupling constant product $\left(g_{K \rho K} G_{N \rho N^{*}}\right)^{2} / 16 \pi^{2} \simeq 40$. The curve shown in Fig. 16 was calculated with this parameter as 56 instead of 40 , a difference easily within the uncertainties of the theoretical estimate.

Because this is a vector-exchange process, the theoretical cross section increases with energy (the calculated cross section is roughly $0.4 \mathrm{mb}$ at $2 \mathrm{GeV} / c, 0.9 \mathrm{mb}$ at $3 \mathrm{GeV} / c$, and $1.1 \mathrm{mb}$ at $5 \mathrm{GeV} / c$ ) in strong disagreement with experiment (see Table II of Ref. 27 and Table I of Ref. 40). The significance of the coupling constants quoted above is therefore obscure.

It is of interest while considering this reaction (where a single-exchange mechanism occurs) to examine the effects of including an intrinsic form factor in the Born amplitude $\left\langle\lambda_{c} \lambda_{d}\left|B_{j}\right| \lambda_{a} \lambda_{b}\right\rangle$ of Eq. (1). There can be no doubt that such structure factors exist, with variations in $\Delta^{2}$ roughly characteristic of the masses of the particles involved. Such $\Delta^{2}$ dependences are unlikely to produce the collimation attributed here to absorption, but their effects need to be explored. The result of including a modest intrinsic form factor, $F\left(\Delta^{2}\right)=M^{2} /\left(\Delta^{2}+M^{2}\right)$ with $M \simeq m_{N}=0.9 \mathrm{GeV}$, is shown by the dashed curves in Figs. 16 and 17. The cross section shows an expected increase in its peaking at small angles, while the decay correlation parameters tend to shift towards the values given by the peripheral model without absorption. The latter fact can be understood from the presence of more partial waves in the Born amplitudes with the form factor than without. Since the absorption depletes a fixed number of the low partial waves, less multilation of the amplitudes occurs with than without the form factor and the resulting decay parameters are less altered.

The changes produced by a relatively slowly varying form factor are of the same order of magnitude as arise from variations of the final state absorption parameters (see Fig. 2). It is clear that until the model is refined well beyond its present state nothing very definite can be said about the presence or absence of intrinsic form factors, unless in a particular process they happen, by some set of circumstances, to vary quite rapidly with $\Delta^{2}$.

$$
\text { B. } K^{-} p \rightarrow \pi^{-} Y^{*}(1385)
$$

In view of the success of the Stodolsky-Sakurai $M 1$ coupling model for $\rho$ exchange in the process $K^{+} p \rightarrow K^{0} N^{*}$, the analogous reaction, $K^{-} p \rightarrow \pi^{-} Y_{1}^{*}$ (1385), is of considerable interest. Here $K^{*}$ exchange is the only simple mechanism. On the basis of unitary

\footnotetext{
${ }^{38}$ In this connection we remark that the off-diagonal elements
of the density matrix shown in Fig. 17 must vanish at $\theta=0$ if the production cross section is finite there. The data do not appear to conform with this very general requirement.
}

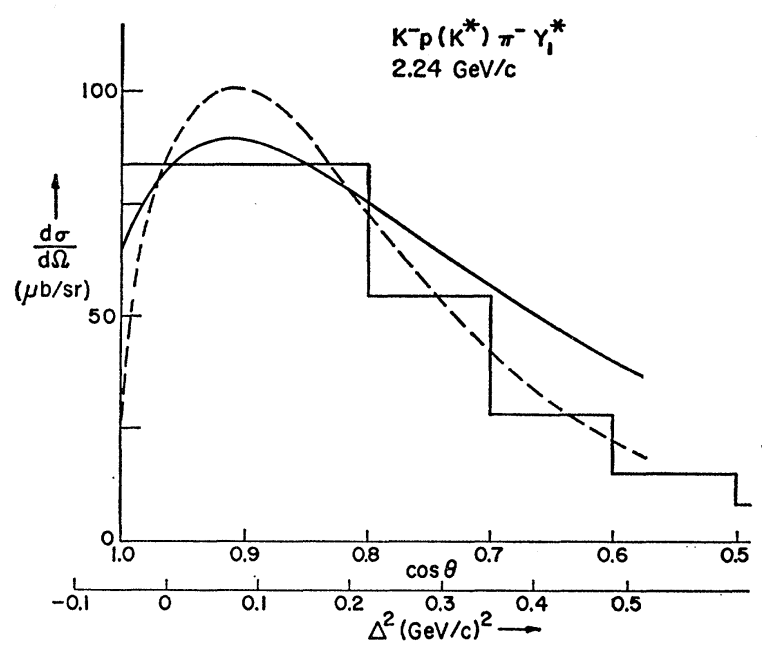

Fig. 18, Differential cross section for the reaction $K^{-} p \rightarrow \pi^{-} Y^{*}$ (1385) at $2.24 \mathrm{GeV} / c$. The preliminary data are from Ref. 39. The solid curve is calculated from the relativistic magnetic dipole coupling model of $K^{*}$ exchange with absorption parameters $C_{+}=0.70, \gamma_{+}=0.077$. The dashed curve includes the intrinsic form factor of Fig. 16 .

symmetry it is expected that the relativistic $M 1$ coupling will be applicable at the $p K^{*} Y^{*}$ vertex, with the resultant well-known decay correlation. Preliminary results $^{39}$ at $2.24 \mathrm{GeV} / c$ confirm this expectation. A comparison of the present theory using the $M 1$ coupling and experiment is shown in Figs. 18 and 19. Just as in Figs. 16 and 17, two sets of curves are shown, one with point couplings in the Born amplitude and one with an intrinsic form factor, $F\left(\Delta^{2}\right)=M^{2} /\left(\Delta^{2}+M^{2}\right), M=0.9$ $\mathrm{GeV} / c$. For both curves the shape of the differential cross section is in adequate agreement with the data. The $Y^{*}$ decay correlation parameters are changed appreciably by the form factor. The explanation is the same as that given at the end of Sec. IVA, with the additional observation that, if anything, there are fewer partial waves present here and the modifications therefore somewhat larger. The theoretical decay correlation parameters, averaged over the interval 0.6 $\leq \cos \theta \leq 1$, are $\left\langle\rho_{33}\right\rangle=0.25(0.30), \operatorname{Re}\left\langle\rho_{3,-1}\right\rangle=0.15(0.23)$, $\operatorname{Re}\left\langle\rho_{3,1}\right\rangle=-0.14(-0.008)$, where the numbers in parentheses are the values with the form factor. For comparison the simple Stodolsky-Sakurai predictions are $\rho_{33}=0.375, \operatorname{Re}_{3,-1}=0.217, \operatorname{Re} \rho_{3,1}=0$, independent of production angle. The experimental results for the first two parameters are $\left\langle\rho_{33}\right\rangle=0.31 \pm 0.05$ and $\operatorname{Re}\left\langle\rho_{3,-1}\right\rangle$ $=0.27 \pm 0.04, \operatorname{Re}\left\langle\rho_{3,1}\right\rangle=0.032 \pm 0.038$. Reasonable agreement is found with the calculations including the form factor.

The experimental reaction cross section is found to be $182 \pm 16 \mu \mathrm{b}$ at $2.24 \mathrm{GeV} / c$, with the contribution from

${ }^{39}$ J. Leitner, H. Primer, M. Goldberg, M. Gundzik, T. Kikuchi, E. L. Hart, K. W. Lai, G. W. London, N. P. Samios, and S. S. Yamamoto, Bull. Am. Phys. Soc. 10, 90 (1965); also private communication. 


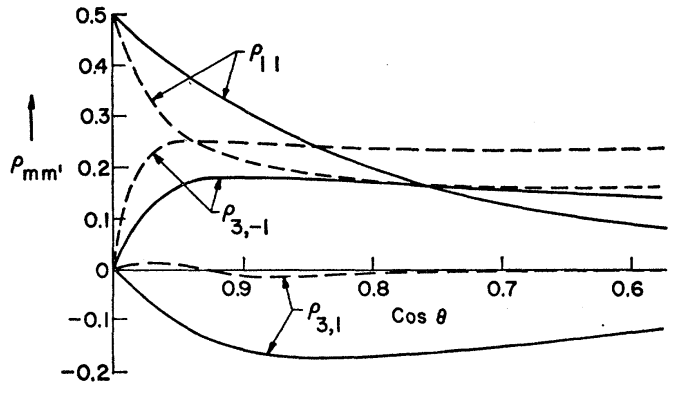

Fig. 19. Elements of the $Y^{*}$ density matrix for the reaction $K^{-} p \rightarrow \pi^{-} Y^{*}(1385)$ at $2.24 \mathrm{GeV} / c$ as functions of production angle. The solid and dashed curves have the same meaning as in Fig. 18. Note that $\rho_{11}+\rho_{33}=0.5$.

the region $\cos \theta>0.6$ being $160 \mu \mathrm{b}$. The $p K^{* 0} Y_{1}{ }^{*+} M 1$ coupling strength ${ }^{22}$ necessary to reproduce this magnitude is $G_{1}^{2} / 4 \pi=50$ and 40 for the point coupling and form factor calculations, respectively. This value may be contrasted with the $S U_{3}$ prediction that the $p K^{* 0} Y_{1}{ }^{*+}$ coupling $G_{1}{ }^{2} / 4 \pi$ should be $\frac{1}{3}$ that for $p \rho^{-} N^{*++}$, the latter inferred from results of Sec. IV.A at $3 \mathrm{GeV} / c$ to be roughly $56 .{ }^{40}$

$$
\text { C. } \pi^{+} p \rightarrow \pi^{0} N^{*}
$$

The production of isobars in $\pi N$ collisions is presumably closely related to the reactions $K^{+} p \rightarrow K^{0} N^{*}$ and $K^{-} p \rightarrow \pi^{-} Y^{*}$,discussed in Secs. IV A and IV B. The peripheral mechanism is $\rho$ exchange and the theoretical differential cross sections and decay correlations are expected to be similar to those for $K^{+} p \rightarrow K^{0} N^{*}$, with allowance for different absorptive effects. The data at $3.54 \mathrm{GeV} / c,{ }^{41}$ as well as at ${ }^{12,42} 2.75$ and $4.0 \mathrm{GeV} / c{ }^{15} \mathrm{do}$ not confirm these expectations. While the decay correlalation data are fitted reasonably well by the StodolskySakurai or the present predictions, the experimental production angular distributions are far more peripheral than the theoretical calculations and the data for $K^{+} p \rightarrow K^{0} N^{*}$. This is shown in Fig. 20, where the 2.75- and $4.0-\mathrm{GeV} / c$ data on $\pi^{+} p \rightarrow \pi^{0} N^{*}$ are compared with the $3.0-\mathrm{GeV} / c$ results for $K^{+} p \rightarrow K^{0} N^{*}$ and with a theoretical curve appropriate to the $4.0-\mathrm{GeV} / c \pi^{+} p$ data. The theoretical cross section is of roughly the

${ }^{40}$ This estimate depends upon the unitary symmetry prediction, $g^{2} K^{+} \rho^{-} K^{0} / 4 \pi=1.0$, whereas in the present reaction the $K^{-} K^{* 0} \pi^{-}$ coupling is known from the $K^{*}$ width to be $g^{2} K^{-} K^{* 0} \pi^{-} / 4 \pi=1.5$. The discrepancy probably lies as much in the incorrect energy dependence of vector-exchange amplitudes as in a failure of unitary symmetry. Evidence for this is found in the fact that the present model is consistent with preliminary data at $3.0 \mathrm{GeV} / c$ [J. Badier, M. Demoulin, J. Goldberg, B. P. Gregory, P. Krejbich, C. Pelletier, M. Ville, R. Barloutaud, A. Leveque, C. Louedec, J. Meyer, P. Schlein, A. Verglas, E. S. Gelsema, J. Hoogland, J. C. Kluyver, and A. G. Tenner, in Proceedings of the 12th Annual Conference on High-Energy Physics, Dubna, 1964 (Moscow, 1965) ], with a cross section of $100 \mu \mathrm{b}$, using the $S U_{3}$ prediction of couplings determined at the same energy.

${ }^{41}$ M. Abolins, D. D. Carmony, D.-N. Hoa, R. L. Lander, C. Rindfleisch, and N.-H. Xuong, Phys. Rev. 136, B195 (1964).

${ }^{42}$ Saclay-Orsay-Bologna-Bari Collaboration, Phys. Letters 13, 341 (1964). correct magnitude at small momentum transfers, but is much too broad. Wide variations of the final-state absorption parameters $C_{-}$and $\gamma_{-}$do not produce large changes in the shape of the differential cross section, but only in its magnitude (see the remarks at the end of Sec. II.A). With $C_{-}=1.0, \gamma_{-}=0.5 \gamma_{+}$and $G^{2}{ }_{1} / 4 \pi=50$, as in Fig. 20, the calculated total cross sections are 0.42 $\mathrm{mb}$ at $2.75 \mathrm{GeV} / c$ and $0.68 \mathrm{mb}$ at $4.0 \mathrm{GeV} / c$, compared with the experimental values of ${ }^{42} 0.30 \pm 0.03 \mathrm{mb}$ and $0.29 \pm 0.03 \mathrm{mb},{ }^{15}$ respectively. The model therefore fails completely to describe the process $\pi^{+} p \rightarrow \pi^{0} N^{*}$ at any energy.

The incorrect energy dependence of the cross section is by now a familiar deficiency of vector-meson-exchange processes, but the marked difference between $\pi^{+} p \rightarrow$ $\pi^{0} N^{*}$ and $K^{+} p \rightarrow K^{0} N^{*}$ is another matter. One possibility is the presence of very different intrinsic form factors as the $\pi \pi \rho$ and $K K \rho$ vertices, but this runs contrary to any notion of approximate $S U_{3}$ symmetry. Another possibility is the removal of $\pi^{+} p$ events from the $N^{*}$ band by final state interactions not included in the diffractive scattering of the model. This effect can be expected to be most important for (a) short-lived resonances like $N^{*}$, (b) large momentum transfers that correspond to close collisions, (c) final states in which the particle(s) other than the resonance interact strongly with it (e.g., $\pi^{0} N^{*}$, rather than $K^{0} N^{*}$ ).

$$
\text { V. } \pi^{+} n \rightarrow \omega p
$$

An interesting example of the production of a vector meson is the reaction $\pi^{+} n \rightarrow \omega p$. The only simple ex-

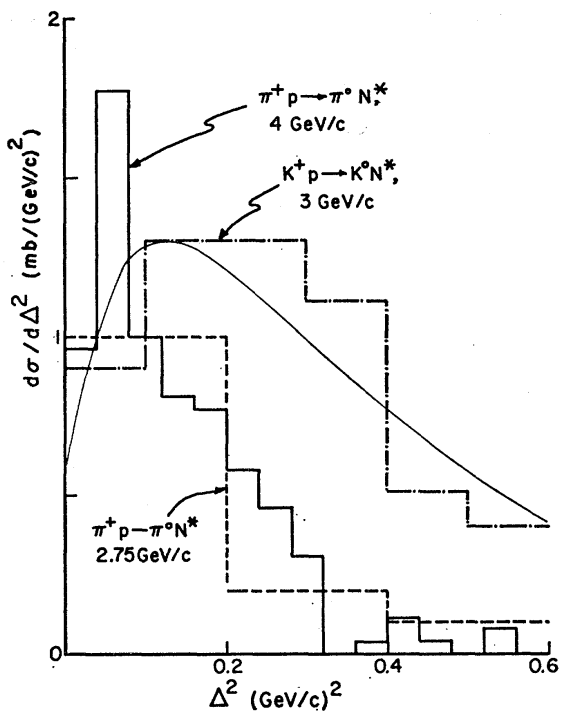

FIG. 20. Comparison of differential cross sections for the reactions $\pi^{+} p \rightarrow \pi^{0} N^{*}$ and $K^{+} p \rightarrow K^{0} N^{*}$. The $4 \mathrm{GeV} / c \pi^{+} p$ histogram from the data of Ref. 15 is normalized properly, but the 2.75 $\mathrm{GeV} / c \pi^{+} p$ data (Ref. 42 ) and the $K^{+} p$ data at $3 \mathrm{GeV} / \mathrm{c}$ (Ref. 25 and Fig. 16) are arbitrary in scale. The curve is calculated for $\pi^{+} p \rightarrow \pi^{0} N^{*}$ at $4 \mathrm{GeV} / c$ assuming $\rho$-exchange and with absorption parameters $C_{+}=0.76, \gamma_{+}=0.040, C_{-}=1.0, \gamma_{-}=0.020$ and magnetic dipole coupling constant $\left(G^{2}{ }_{1}\right)_{p \rho^{+} N^{*}} / 4 \pi=50$. 


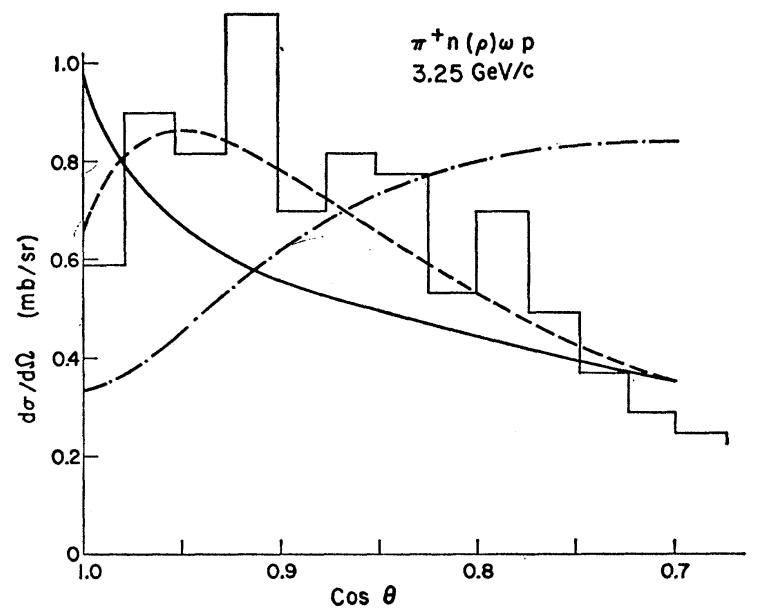

Frg. 21. Comparison of theoretical and experimental differential cross sections for $\pi^{+} n \rightarrow \omega p$ at $3.25 \mathrm{GeV} / c$. The data, shown with arbitrary normalization, are from Ref. 44 . The coupling constants used in the calculations are $f^{2}{ }_{\pi \rho \omega} / 4 \pi=10,\left(G^{V}{ }_{n \rho}{ }^{+}\right)^{2} / 4 \pi=2.0$. The absorption parameters are $C_{+}=0.80, \gamma_{+}=0.046, \gamma_{-}=0.034$. The solid and dashed curves both have $C_{-}=1$, but correspond to $G^{T} / G^{V}=3.7$ and 2.0 , respectively. The dot-dashed curve has $C_{-}=0.9$ and $G^{T} / G^{V}=3.7$.

change process allowed by isospin and $G$ parity is $\rho$ exchange. But the decay correlation data at ${ }^{43} 1.7$ and $^{44} 3.25 \mathrm{GeV} / c$ imply $\left\langle\rho_{00}\right\rangle>0.5$, a value completely inconsistent with the prediction $\left(\rho_{00}=0\right)$ of the simple $\rho$-exchange model without absorption.

The results of calculations with the present model at $3.25 \mathrm{GeV} / c$ are compared with experiment ${ }^{44}$ in Figs. 21 and 22. The first observation to be made is that anything but complete absorption of the lowest partial waves $\left(C_{+}\right.$and/or $\left.C_{-}=1\right)$, as typified by the dot-dashed curve in Fig. 21, is in gross disagreement with the experimental differential cross section. The other curves show the effects of variation of the ratio $G^{T} / G^{V}$, the solid curve corresponding to the couplings of the $\rho$ meson inferred from the isovector electromagnetic form factors. The experimental data on the angular distribution (Fig. 21) and decay paramenters (Fig. 22) agree tolerably with the "true" $\rho$-exchange curve, provided $C_{-}=1.45$ Similar agreement occurs at $1.7 \mathrm{GeV} / c$ (even though this momentum is probably too low for applicability of our model) for both the shape of the cross section for $\cos \theta>0.7$ and the decay correlations. The experimental (theoretical) density-matrix elements, averaged over the interval $1 \geq \cos \theta \geq 0.7$, are $\left\langle\rho_{00}\right\rangle=0.60 \pm 0.12(0.62)$, $\left\langle\rho_{1,-1}\right\rangle=0.0 \pm 0.712(0.05),\left\langle\operatorname{Re}_{10}\right\rangle=-0.06 \pm 0.08(-0.11)$. The agreement is even better than at $3.25 \mathrm{GeV} / c$.

This reaction is a striking example of the differences

${ }^{43}$ T. C. Bacon, W. J. Fickinger, D. G. Hill, H. W. K. Hopkins, D. K. Robinson, and E. O. Salant, Bull. Am. Phys. Soc. 10, 66 (1965); and private communication.

${ }^{44}$ H. O. Cohn, W. M. Bugg, and G. T. Condo, Phys. Letters 15, 344 (1965).

${ }_{45}$ An even larger value of $G^{T} / G^{V}$ is indicated by the experimental value of $\rho_{00}$, but it is difficult to increase $\rho_{00}$ above 0.6 , whatever the ratio $G^{T} / G^{V}$. that can occur between the peripheral models with and without absorption. The observed decay correlations do not even resemble qualitatively those of the peripheral model without absorption, assuming $\rho$ exchange. One may in fact ask, on the basis of past experience with absorptive effects, why there is such a dramatic change in the decay correlations here when absorption is included. In $\rho$ production with pion exchange, for example, there are some changes, but the gross feature of a large $\rho_{00}$ and small $\rho_{1,-1}$ persists. The reason for the modest effects of absorption in $\rho$ production and the radical effects in the present reaction can be understood in part as follows: At a given energy, the lighter the mass of the exchanged particle, the more partial waves that enter significantly; and for a fixed mass, the higher the energy, the more partial waves. The decay-correlation parameters depend on a delicate combining of the various helicity amplitudes, each with its partial-wave expansion. Furthermore, the deep minimum of the differential cross section in the forward direction for $\omega$ production in the simple $\rho$-exchange model [see Eq. (14) of Ref. 22] depends on a cancellation at $\cos \theta=1$ of "normal" partial-wave expansions and anomalous terms in the lowest partial waves $\left[A_{n}{ }^{(k)}\right.$ and $\bar{A}_{n}{ }^{(k)}$ terms in Eq. (6)]. When absorption of the lowest partial waves is introduced, the delicate balances and cancellations are destroyed-the cross section peaks at $\cos \theta=1$ instead of falling towards zero; the densitymatrix elements are altered. When the number of partial waves is relatively small, the absorption produces drastic effects. ${ }^{46}$

In spite of the success of our model in giving decay

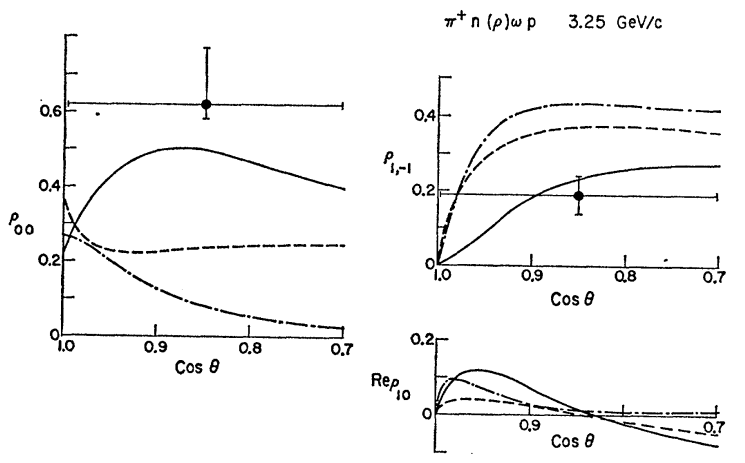

FIG. 22. Theoretical and experimental $\omega$ density matrix elements for $\pi^{+} n \rightarrow \omega p$ at $3.25 \mathrm{GeV} / c$. The meaning of the curves is the same as in Fig. 21.

46 The relative size of the changes caused by absorption in pionexchange processes and the present reaction can be understood in these terms. But one might ask about the other $\rho^{-}$and $K^{*}$-exchange processes, such as the isobar production reactions of Sec. IV, in which the Stodolsky-Sakurai predictions fit the data. The answer is that there are sizeable changes there (see Fig. 17), but the average values of the density-matrix elements happen to be of the same general magnitude as those of the simple model. The quantitative effects of absorption depend in detail on the spins and parities of the particles involved. 
correlations and shapes of differential cross sections for $\pi^{+} n \rightarrow \omega p$, it fails again on the energy dependence. With the coupling constants of Fig. 21 and $G^{T} / G^{V}=3.7$, the calculated cross sections (for $\cos \theta>0.7$ ) at 1.7 and 3.25 $\mathrm{GeV} / c$ are 0.20 and $1.0 \mathrm{mb}$, respectively. At $1.7 \mathrm{GeV} / c$ the observed total cross section is $1.68 \pm 0.19 \mathrm{mb}^{43}$ with $0.4 \mathrm{mb}$ coming from $\cos \theta>0.7$, while at $3.25 \mathrm{GeV} / c$ a comparison of the reactions $\pi^{+} n \rightarrow \omega p$ and $\pi^{+} n \rightarrow \rho^{0} p$ allows one to estimate from Fig. 7 that the total cross section is approximately $0.2 \mathrm{mb}$.

\section{DOUBLE RESONANCE PRODUCTION}

In four- and five-body final states the formation of two resonances $\left(\rho N^{*}, K^{*} N^{*}, \omega N^{*}\right)$ is a prominent feature at all incident momenta. We discuss here the reactions, $\pi^{+} p \rightarrow \rho^{0} N^{*}$ and $K^{+} p \rightarrow K^{*} N^{*}$, where the dominant mechanism is pion exchange. The process $\pi^{+} p \rightarrow \omega N^{*}$, with $\rho$ exchange as the only simple possibility, has been treated elsewhere. ${ }^{47}$

If pion exchange is taken as the only mechanism, there are no adjustable coupling constants at our disposal. Because there are two unstable particles in the final state the calculations were performed with a rather wide range of final-state absorption parameters. The conclusion from this exploration is that, while the shape of the differential cross section and the decay correlation data are well reproduced by the theory with reasonable choices of $C_{-}$and $\gamma_{-}$, the magnitudes of the theoretical cross sections are too large by a factor of 2-3 at incident momenta of $3-4 \mathrm{GeV} / c^{48}$ The specific areas of agreement and disagreement are described below, as well as a reason for the failure of the model to give the absolute cross sections correctly.

$$
\text { A. } \pi^{+} p \rightarrow \varrho^{0} N^{*}
$$

Data are available on the production of $\rho N^{*}$ in $\pi^{+} p$ collisions at $2.77,{ }^{49} 3.65,{ }^{50} 4.0,{ }^{, 51}$ and $8.0 \mathrm{GeV} / c^{17} \mathrm{~A}$ comparison of theory and experiment at $4 \mathrm{GeV} / c$ has already been given in Figs. 13 and 14 of Ref. 7. Similar agreements are found at $3.65 \mathrm{GeV} / c$. The shape of the differential cross section is reproduced reasonably well and the calculated decay-correlation parameters are in embarassingly good agreement with the data ${ }^{50}$ : The

${ }^{47}$ B. E. Y. Svensson, Nuovo Cimento (to be published). In this reaction, generally satisfactory agreement with the data is obtained. In particular, the theory is able to reproduce the very significant departure of some of the density matrix elements from the values given by the simple $\rho$-exchange model without absorption, just as in Sec. V for $\pi^{+} n \rightarrow \omega p$.

${ }^{48}$ In these calculations we use a value of $\gamma_{-}$that is of the same order of magnitude as that obtained from the elastic-scattering data (i.e., $0.25<\gamma_{-} / \gamma_{+}<1.0$ ), and $C_{-}$in the range $0.5<C_{-} \leq 1.0$. One might hope to remove this disagreement by assuming a finalstate interaction between the resonances that is completely different from that in the incident channel. This hope is not borne out by detailed calculations. If we allow $\gamma_{-}$and $C_{-}$free rein, we are able to achieve the desired reduction of the cross section, but only at the cost of spoiling the shape of the angular distribution.

${ }^{49} \mathrm{~S}$. S. Yamamoto, J. R. Smith, D. C. Rahm, and J. J. Lloyd, in Proceedings of the 12th Annual International Conference on High-Energy Physics, Dubna, 1964 (Moscow, 1965). experimental (theoretical) values for the $\rho$ meson's density-matrix elements, averaged over $0<\Delta^{2}<20 \mu^{2}$, are

$$
\begin{gathered}
\left\langle\rho_{00}\right\rangle=0.72 \pm 0.04(0.74),\left\langle\rho_{1,-1}\right\rangle=-0.003 \pm 0.04(0.02) \\
\left\langle\operatorname{Re} \rho_{10}\right\rangle=-0.044 \pm 0.03(-0.06)
\end{gathered}
$$

The $N^{*}$ density-matrix elements are

$$
\begin{aligned}
\left\langle\rho_{11}\right\rangle & =0.45 \pm 0.03(0.42), \\
\left\langle\operatorname{Re} \rho_{3,-1}\right\rangle & =0.013 \pm 0.03(0.006), \\
\left\langle\operatorname{Re} \rho_{31}\right\rangle & =-0.045 \pm 0.04(-0.04) .
\end{aligned}
$$

While there is some question on the experimental value of the total cross section around $4 \mathrm{GeV} / c$ (at 3.65 $\mathrm{GeV} / c$, the value quoted is $1.17 \pm 0.12 \mathrm{mb}^{50}$; at 4.0 $\mathrm{GeV} / c$ it is given as $\left.{ }^{51} 0.60 \pm 0.18 \mathrm{mb}\right)$, it is much smaller than our calculated $2 \mathrm{mb}$ (using $\gamma_{+}=0.040, C_{+}=0.76$, $\left.\gamma_{-}=0.020, C_{-}=1.0\right)$. At $8 \mathrm{GeV} / c$ the comparison is more favorable. The experimental value can be estimated from Ref. 17 to be $0.6-0.7 \mathrm{mb}$ and the theoretical result is $0.8 \mathrm{mb}$ (for $\gamma_{-}=0.5 \gamma_{+}, C_{-}=1.0$ ).

\section{B. Combined Correlations in $\pi^{+} p \rightarrow \varrho^{0} N^{*}$}

The excellent agreement between theory and experiment on the separate decay correlations for $\rho^{0}$ and $N^{*}$ at 3.65 and $4.0 \mathrm{GeV} / c$ argues strongly for the validity of pion exchange as the dominant mechanism. In addition to the individual decay distributions, data on combined correlation effects are presented in Ref. 50. Significant changes were found in the $N^{*}$ and $\rho$ decay angular distributions when selection was made of different angular intervals in the other resonance's decay. On the basis of the simple peripheral model without absorption such combined correlations cannot occur for pion exchange, but one might expect that absorptive effects (which cause deviations of the individual density matrices from the predictions of the simple model) could produce such correlations.

In a two-body reaction, $a b \rightarrow c d$, where $c$ and $d$ are both unstable, advantage can be taken of the Lorentz transformation properties of helicity amplitudes ${ }^{52}$ to obtain a distribution describing simultaneously the decay of $c$ in its rest frame and the decay of $d$ in its rest frame ${ }^{53}$ :

$$
\begin{aligned}
W\left(\theta_{c}, \varphi_{c} ; \theta_{d}, \varphi_{d}\right)= & N \sum_{m_{c} m^{\prime} c} \sum_{m_{d} m^{\prime} d}\left\langle m_{c} m_{d}|\rho| m_{c}^{\prime} m_{d}^{\prime}\right\rangle \\
& \times X^{s_{c} m_{m_{c} m^{\prime} c}}\left(\theta_{c}, \varphi_{c}\right) X^{s_{d}{ }_{m_{d} m^{\prime} d}}\left(\theta_{d}, \varphi_{d}\right),
\end{aligned}
$$

${ }^{50} \mathrm{G}$. Goldhaber, "Multipion and baryon resonances in the $\pi^{+} p$ reaction," Proceedings of the Conference on Particle and High-Energy Physics, Boulder, Colorado, 1964 (to be published); and (private communication).

51 Aachen-Berlin-Birmingham-Bonn-Hamburg-London-München Collaboration, Nuovo Cimento 35, 659 (1965).

${ }_{52}$ The key relation for the derivation is Eq. (6) of Ref. 18 which contains the fact that as one goes from the rest frame of $c$ to the rest frame of $d$ only the helicities of $a$ and $b$ change. The subsequent derivation follows closely that of Sec. 3 of Ref. 18 .

${ }^{53}$ An independent derivation of this result has been given by H. Pilkuhn and B. E. Y. Svensson, Nuovo Cimento (to be published). 


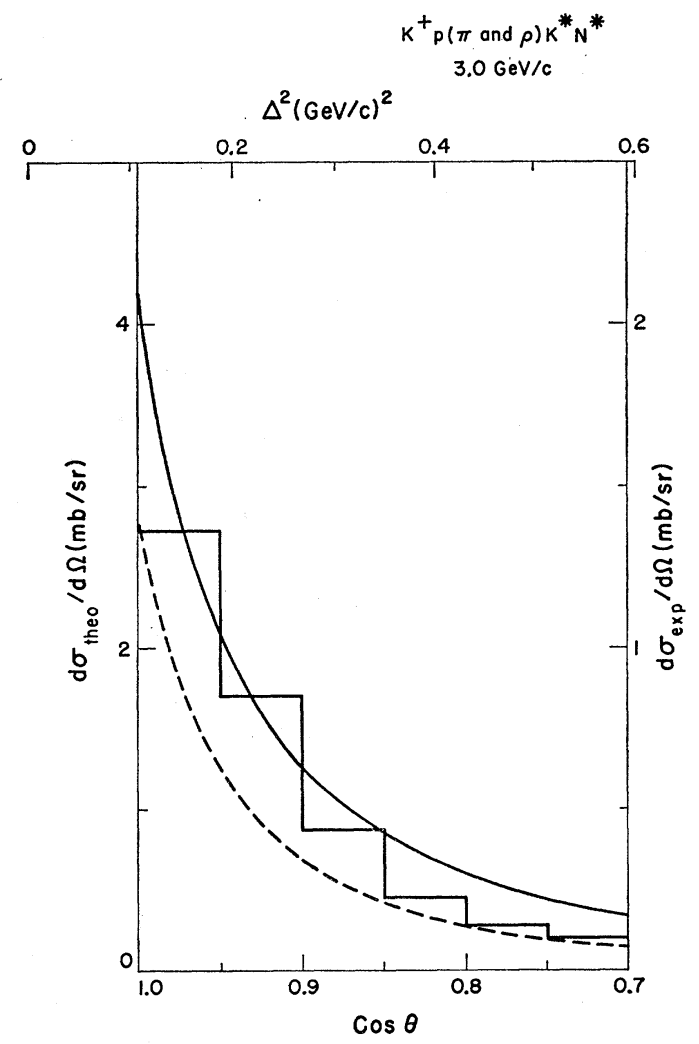

Fig. 23. Differential cross section for the reaction $K^{+} p \rightarrow K^{*} N^{*}$ $\left(K^{+} \pi^{-} \pi^{+} p\right)$ at $3.0 \mathrm{GeV} / c$. The data are from Ref. 56. The absorption parameters are $\gamma_{+}=0.110, \gamma_{-}=0.055, C_{+}=0.9, C_{-}=1.0$. The solid curve represents pion exchange only, with known coupling constants, $g^{2} K^{+} \pi^{-} K^{* 0} / 4 \pi=1.5,\left(G^{*} \pi^{+} p N^{*++}\right)^{2} / 4 \pi=0.43$. The dashed curve includes $\rho$ exchange with coupling constants, $f^{2} K^{+} \rho^{-} K^{* 0} / 4 \pi$ $=5, G^{2} 1 / 4 \pi=56$. Note that the right (left) ordinate scale applies to the experimental (theoretical) cross section.

where, for the decays of interest here, ${ }^{54}$

$$
X_{m m^{\prime}}^{s}(\theta, \varphi)=\sum_{\lambda \lambda^{\prime}} e^{i\left(m-m^{\prime}\right) \varphi} d_{m \Lambda}^{s}(\theta) d^{s}{ }_{m^{\prime} \Lambda}(\theta) .
$$

In (10), $\Lambda=\lambda-\lambda^{\prime}, \lambda, \lambda^{\prime}$ being the helicities of the two decay products from the resonance. The form of the combined density matrix $\left\langle m_{c} m_{d}|\rho| m^{\prime}{ }_{c} m_{d}^{\prime}\right\rangle$ depends on the coordinate systems chosen for the decays. We make the standard choice of the $y$ axis as the normal to the production plane $(\mathbf{n}=\mathbf{a} \times \mathbf{c})$ and the $z$ axis for the decay of $c$ as the direction of $a$, as seen in the rest frame of $c$, and the $z$ axis for the decay of $d$ as the direction of $b$, as seen in the rest frame of $d$. Then the combined density matrix can be expressed in terms of the c.m. helicity amplitudes as

$$
\begin{aligned}
& \left\langle m_{c} m_{d}|\rho| m^{\prime}{ }_{c} m^{\prime}{ }_{d}\right\rangle=\sum_{\text {all } \lambda} d^{s_{c}}{ }_{m_{c} \lambda_{c}}\left(\psi_{c}\right) d^{s_{d}{ }_{m_{d} \lambda_{d}}}\left(\psi_{d}\right) \\
& \times\left\langle\lambda_{c} \lambda_{d}|M| \lambda_{a} \lambda_{b}\right\rangle\left\langle\lambda^{\prime}{ }_{c} \lambda_{d}^{\prime}|M| \lambda_{a} \lambda_{b}\right\rangle^{*} \\
& \chi d^{s c}{ }_{m^{\prime}{ }_{c}{ }^{\prime}{ }_{c}}\left(\psi_{c}\right) d^{s{ }{ }_{m^{\prime}}{ }_{d \lambda^{\prime}}{ }_{d}}\left(\psi_{d}\right),
\end{aligned}
$$

${ }^{54}$ For more general decays, $X=\Sigma_{\lambda \alpha \lambda \beta} A_{m} A^{*}{ }_{m}$ ', where $A_{m}$ is given by Eq. (15) of Ref. 18 . where $\psi_{c}, \psi_{d}$ are the angles of rotation from the helicity directions to the $z$ axes (see Refs. 18 and 53). Integration over $\left(\theta_{c}, \varphi_{c}\right)$ yields the individual decay correlations $^{18}$ for $d$, and vice versa. In the experiment at 3.65 $\mathrm{GeV} / c,{ }^{50}$ averages over the two azimuths $\varphi_{c}$ and $\varphi_{d}$ are performed. The relevant decay distribution then involves only the diagonal elements of the combined density matrix.

Before comparing theoretical results with the data some qualitative observations are in order. Let $c$ be the $\rho^{0}$ and $d$ be the $N^{*}$. If $\rho^{0}$ decays are chosen with $\cos \theta_{c}= \pm 1$, only amplitudes with $m_{c}=0$ contribute, and, for small momentum transfers in production, the familiar Adair analysis implies that the $N^{*}$ decay correlation will be closely $\left(1+3 \cos ^{2} \theta_{d}\right)$, independently of the production mechanism. For $\rho^{\theta}$ decays such that $\cos \theta_{c}=0$, only $m_{c}= \pm 1$ occur and a different (and model-dependent) $N^{*}$ decay distribution is produced. For $N^{*}$ decays with $\cos \theta_{d}= \pm 1$, only the magnetic substates $m_{d}= \pm \frac{1}{2}$ occur, while for $\cos \theta_{d}=0, m_{d}= \pm \frac{3}{2}$ and $\pm \frac{1}{2}$ contribute with relative intensities of $3: 1$, respectively. But the Adair argument does not apply and nothing general can be said about the $\rho^{0}$ decay distributions.

The combined density matrix (11) has been calculated for the reaction $\pi^{+} p \rightarrow \rho^{0} N^{*}$ at $3.65 \mathrm{GeV} / c$ as a function of production angle, assuming pion exchange only. Suitable averages of (9) over momentum transfer $\left(0<\Delta^{2}<20 \mu^{2}\right)$ and decay angles $\left(\left|\cos \theta_{c}\right|>0.4,\left|\cos \theta_{c}\right|\right.$ $<0.4$, and similarly for $\theta_{d}$ ) were made in order to yield results for comparison with the data. ${ }^{50}$ An effect in qualitative and even semiquantitative agreement with experiment is obtained. ${ }^{55}$ For the $N^{*}$ decay distributions, the theoretical expressions are $W_{N}{ }^{*} \sim 1+2.04 \cos ^{2} \theta_{d}$ for

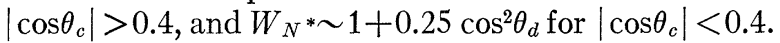
The $\rho^{0}$ decay distributions are $W_{\rho} \sim 1+6.4 \cos ^{2} \theta_{c}$ for $\left|\cos \theta_{d}\right|>0.4$ and $W_{\rho} \sim 1+2.2 \cos ^{2} \theta_{c}$ for $\left|\cos \theta_{d}\right|<0.4$. We see that the present model of pion exchange plus absorption, successful in reproducing the separate $\rho^{0}$ and $N^{*}$ density matrices, also gives a satisfactory description of the existing data on combined correlations.

\section{C. $K^{+} p \rightarrow K^{*} N^{*}$ at 3.0 and $3.5 \mathrm{GeV} / c$}

The production of $K^{*}$ and $N^{*}$ in the final state $\left(K^{+} \pi^{-} \pi^{+} p\right)$ from $K^{+} p$ interactions has been studied at ${ }^{56}$

${ }^{55}$ The experimental results are given in Fig. 16 and Table IV of Ref. 50. The data seem to show a somewhat larger effect than calculated. But results at $4 \mathrm{GeV} / c$ [Aachen-Berlin-BirminghamBonn-Hamburg-London-München Collaboration, Phys. Rev. 138, B897 (1965)] indicate smaller changes. Another complication is the sizable asymmetry seen for both $\rho^{0}$ and $N^{*}$ decays. It seems fair to regard the agreement between theory and experiment as satisfactory, considering the possibility of biases in the experiment, on the one hand, and the idealization of the $\rho^{0}$ and $N^{*}$ as longlived systems with $J=1^{-}$and $J=\frac{3}{2}+$ in the theory on the other.

${ }_{56} \mathrm{M}$. Ferro-Luzzi, R. George, Y. Goldschmidt-Clermont, V. P. Henri, B. Jongejans, D. Leith, G. Lynch, F. Muller, and J.-M. Perreau, Proceedings of the Sienna International Conference on Elementary Particles, 1963, edited by G. Bernadini and G. P. Puppi (Società Italiana di Fisica, Bologna, 1963), Vol. I, p. 189; also (private communication). 
K* DENSITY MATRIX
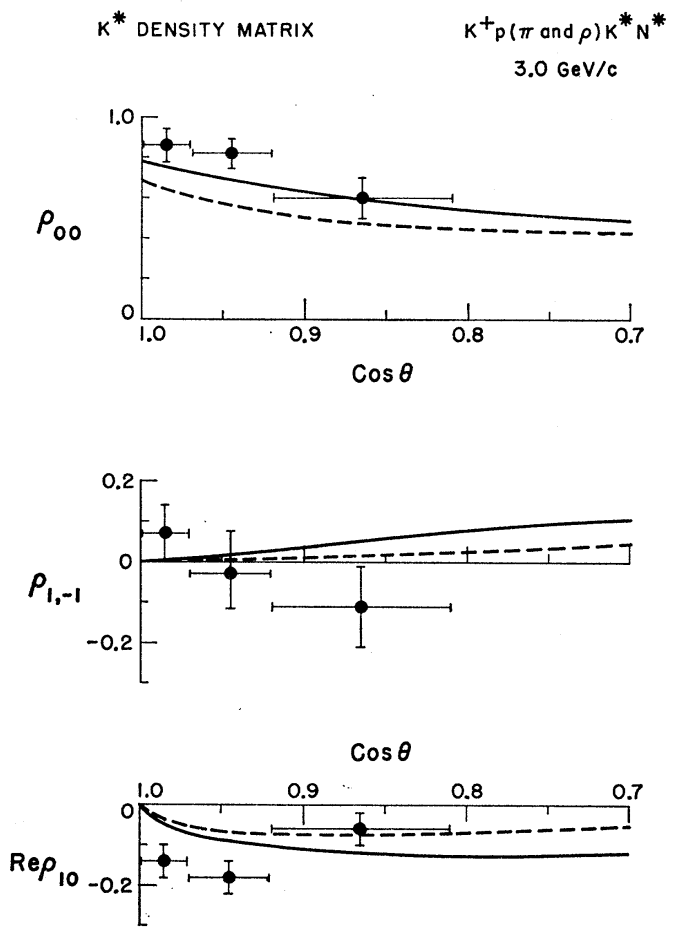

FIG. 24. $K^{*}$ density-matrix elements as functions of production angle for $K^{+} p \rightarrow K^{*} N^{*}$ at $3 \mathrm{GeV} / c$. The data are from Ref. 56. The curves have the same meaning as in Fig. 23.
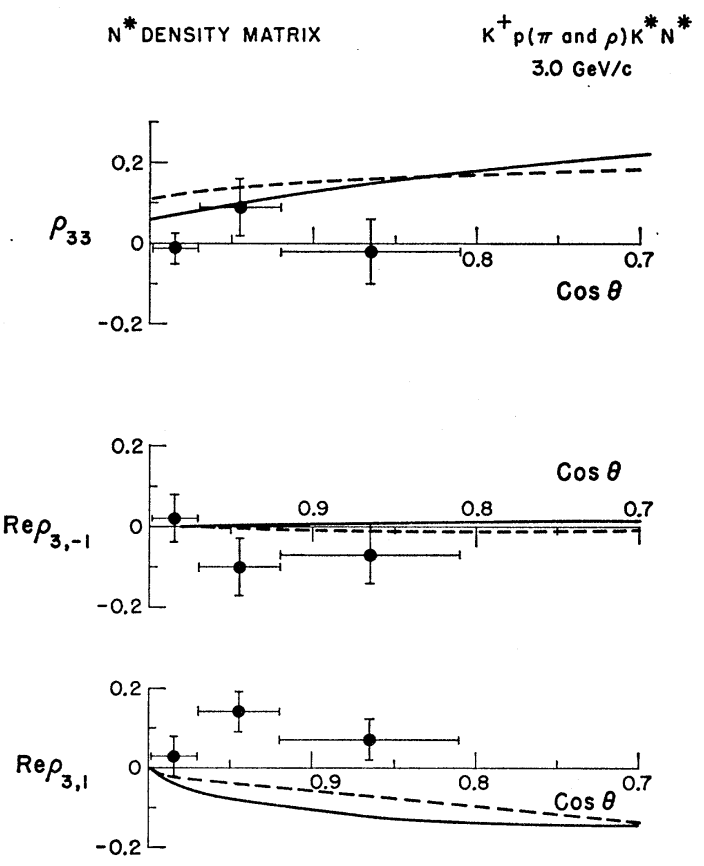

Fig. 25. $N^{*}$ density-matrix elements as functions of production angle for $K^{+} p \rightarrow K^{*} N^{*}$ at $3 \mathrm{GeV} / c$. The data are from Ref. 56 The curves have the same meaning as in Fig. 23.
3.0 and $3.5 \mathrm{GeV} / c .{ }^{57}$ Figures $23-28$ show a comparison between theory and experiment for the differential cross sections and the $K^{*}$ and $N^{*}$ density matrices at these two energies. The calculations have been done with pion exchange only (solid curves) and with a mixture of pion and $\rho$-meson exchange (dashed curves). The strength of the $\rho$-exchange amplitude (whose sign was chosen to give destructive interference) was determined using $S U_{6}$ to relate the $K^{+} \rho^{-} K^{* 0}$ and $\pi \rho \omega$ vertices and taking the $M 1$ coupling for $p \rho N^{*}$ from the

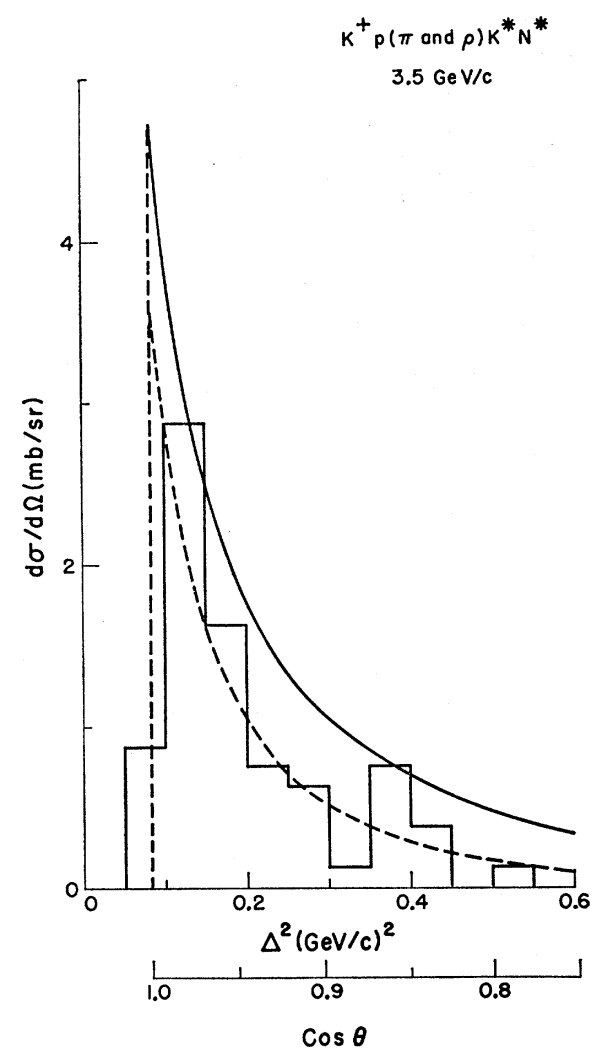

FIG. 26. Differential cross section for the reaction $K^{+} p \rightarrow K^{*} N^{*}$ $\left(K^{+} \pi^{-} \pi^{+} p\right)$ at $3.5 \mathrm{GeV} / c$. The data are from Ref. 57. The meaning of the curves and the coupling constants are the same as in Fig. 23, but the absorption parameters are $\gamma_{+}=0.10, \gamma_{-}=0.05, C_{+}=0.99$, $C_{-}=1.0$.

results on $K^{+} p \rightarrow K^{0} N^{*}$ at the same energy (Sec. IVA).

While the experimental density-matrix elements for $K^{*}$ and $N^{*}$ perhaps agree better with the curves calculated with only pion exchange, no real choice can be made between the different calculations. ${ }^{58}$ Similarly, the shapes of the two theoretical differential cross

${ }^{57}$ F. Grard, J. Debaisieux, J. Heughebaert, L. Pape, and R. Windmolders, in Proceedings of the 12th Annual International Conference on High-Energy Physics, Dubna, 1964 (Moscow, 1965).

${ }^{58}$ Constructive interference between the pion and $\rho$-exchange amplitudes gives decay correlation parameters almost indistinguishable from those calculated with pion exchange alone. 
$K^{*}$ DENSITY MATRIX
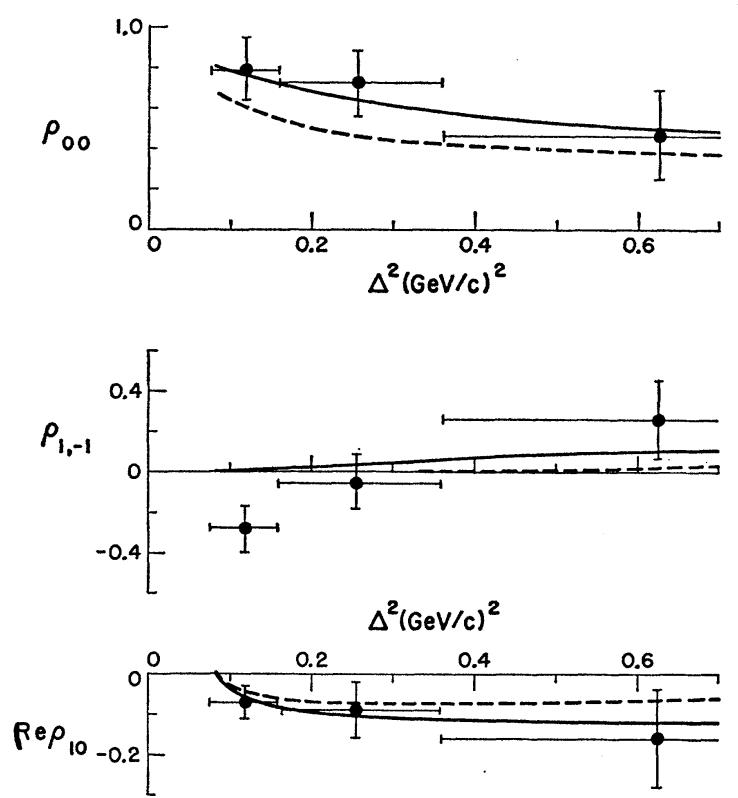

FIG. 27. Same as Fig. 24, but at $3.5 \mathrm{GeV} / c$, with absorption parameters as in Fig. 26. The data are from Ref. 57.

sections are both in reasonable accord with the data. But the destructive interference between the pion and $\rho$-exchange amplitudes causes an appreciable decrease in the absolute value of the cross section. At $3.0 \mathrm{GeV} / c$, the theoretical cross sections for $\cos \theta>0.7$ are $2.3 \mathrm{mb}$ ( $\pi$ exchange only) and $1.2 \mathrm{mb}(\pi$ and $\rho$ ). The experimental cross section for this angular interval is roughly $1.0 \mathrm{mb} .{ }^{56}$ At $3.5 \mathrm{GeV} / c$, the two theoretical values, for $\Delta^{2}<0.6(\mathrm{GeV} / c)^{2}$, are 1.9 and $1.1 \mathrm{mb}$, respectively. Clearly the inclusion of $\rho$ exchange improves the over-all comparison with experiment.

\section{Unitarity Requirements}

Without additional data it is impossible to decide whether the rough agreement in absolute cross section produced by adding $\rho$ exchange to the dominant pion exchange is significant or merely fortuitous. It seems probable that this general feature of too large cross sections in double resonance production can be attributed to the large values of the pertinent coupling constants (i.e., $G^{*}{ }_{\pi N N^{*}}$, etc.) and the absence in the model of any enforcement of the requirements of unitarity. This can be seen by computing the reaction cross section for the various partial waves. For the reaction $K^{+} p \rightarrow K^{*} N^{*}$ at $3 \mathrm{GeV} / c$, Fig. 29 shows a comparison of the partial-wave cross sections with the bound set by unitarity. For all partial waves the cross section, even including absorption, lies uncomfortably close to the bound that represents the total contribution from all

reaction channels, as inferred from the elastic-scattering amplitude. Variations in final-state absorption parameters can change the curve in Fig. 29 somewhat, but it appears that the strength of the exchange force operative in these reactions does not satisfy the criteria on which the distorted-wave Born approximation is based. If this explanation for the discrepancies is correct, models that incorporate unitarity from the beginning ${ }^{59}$ will presumably yield absolute cross sections in better agreement with experiment.

\section{SUMMARY AND DISCUSSION}

(1) The model successfully describes the reaction $\pi N \rightarrow \rho N$ for incident momenta from $\sim 2$ to $8 \mathrm{GeV} / c$ (see Figs. 3-7). The differential cross section, in both shape and absolute value, is well accounted for on the basis of pion exchange alone.

(2) In the $2-3-\mathrm{GeV} / c$ region the model is in agreement with all the data on $K N \rightarrow K^{*} N$, including the $\Delta^{2}$ dependence of the decay correlation parameters (see Figs. 10-14). Vector-meson exchange is necessary in addition to the known amount of pion exchange. The two vector-meson coupling constants are determined from the $K^{+} p \rightarrow K^{*} p$ differential cross section

$N^{*}$ DENSITY MATRIX
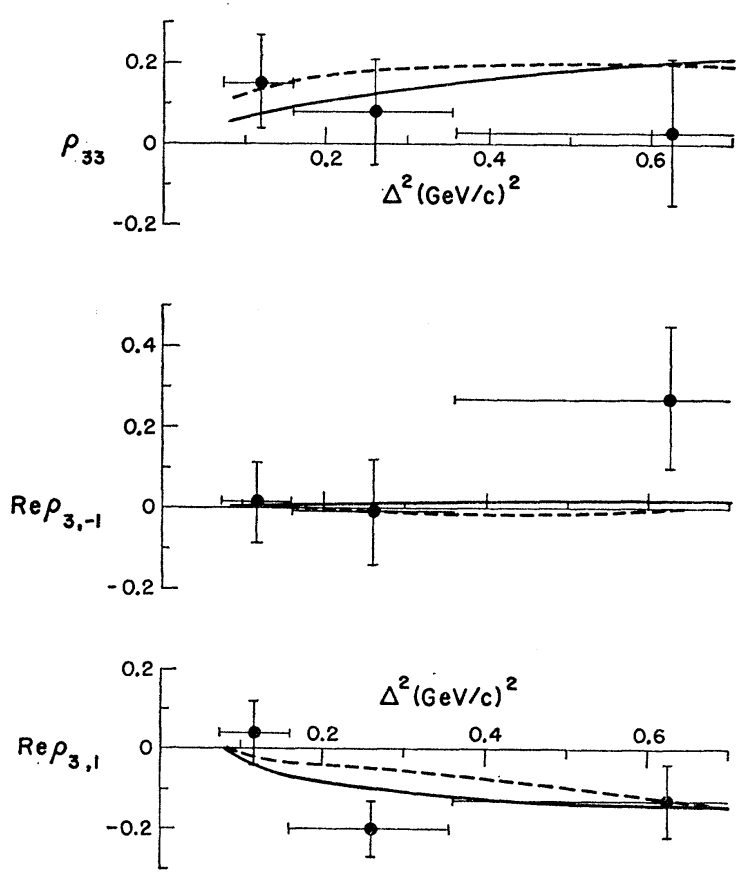

FIG. 28. Same as Fig. 25, but at $3.5 \mathrm{GeV} / c$, with absorption parameters as in Fig. 26. The data are from Ref. 57.

${ }^{59} \mathrm{~K}$. Dietz and H. Pilkuhn, Nuovo Cimento (to be published); D. B. Lichtenberg and P. K. Williams, Phys. Rev. 139, B179 (1965); see also R. C. Arnold, Ref. 4. 


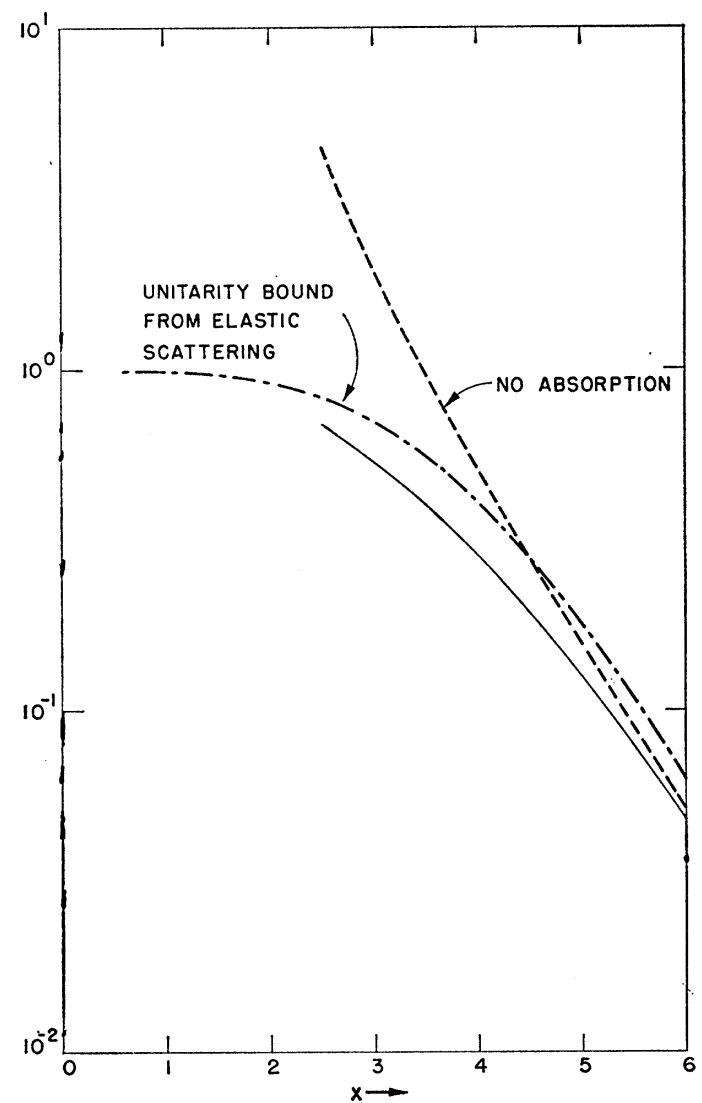

FIG. 29. Partial-wave cross section, divided by the absolute unitarity bound $(2 j+1) \pi / q^{2}$, as a function of $x=j+\frac{1}{2}$ for the reaction $K^{+} p \rightarrow K^{*} N^{*}$ at $3 \mathrm{GeV} / c$. The curves are calculated assuming only pion exchange. The solid (dashed) curve is with (without) absorption, the absorption parameters being $\gamma_{+}=\gamma_{-}$ $=0.11, C_{+}=0.9, C_{-}=1.0$. The dot-dashed curve is the bound inferred from the elastic-scattering data [i.e., $1-\left|e^{2 i \delta+}\right|^{2}$, with $e^{2 i \delta}+$ given by Eq. (4)]. The solid curve is not shown for $x<2.5$ because of ambiguities in the low partial-wave amplitudes [the anomalous terms in Eq. (6)].

at $3 \mathrm{GeV} / c$; two sets of solutions are found (see Fig. 9). The remaining data (i.e., decay correlations and $\left.\bar{K} p \rightarrow \bar{K}^{*} p\right)$ do not distinguish between these.

(3) With the vector-meson coupling constants determined at $3 \mathrm{GeV} / c$, the calculated differential cross section for $K^{+} p \rightarrow K^{*} p$ at $5 \mathrm{GeV} / c$ is much too broad (see Fig. 15). Appreciably smaller values of these coupling constants and/or modification of the vector-meson propagator are necessary to fit the preliminary data. This failure is symptomatic of the model's inability to prevent the well-known increase of vector- and higher spin-exchange amplitudes with energy.

(4) The data on $K^{+} p \rightarrow K^{0} N^{*}$ at $3 \mathrm{GeV} / c$ are well reproduced assuming $\rho$ exchange with magnetic dipole coupling at the $\rho N N^{*}$ vertex (see Figs. 16 and 17). Similarly the available results on $K^{-} p \rightarrow \pi^{-} Y^{*}(1385)$ are adequately described by $K^{*}$ exchange with the same coupling (see Figs. 18 and 19). But here too the vectormeson-exchange mechanism produces an energy de- pendence of the cross section that disagrees with experiment.

(5) The model accounts for neither the very peripheral nature of the process $\pi^{+} p \rightarrow \pi^{0} N^{*}$, nor the rapid decrease of its cross section with increasing energy.

(6) The calculations on $\pi^{+} n \rightarrow \omega p$, assuming $\rho$ exchange, are in reasonable agreement with existing data. The surprisingly large value of $\rho_{00}$ is reproduced by the theory. The peripheral model without absorption gives $\rho_{00}=0$.

(7) The shapes of the differential cross section and the decay correlations for reactions in which two resonances are produced (e.g., $\pi p \rightarrow \rho N^{*}, K N \rightarrow K^{*} N^{*}$ ) are given satisfactorily by the model with reasonable choices of final-state absorption parameters. Of special interest are the combined decay-correlation effects observed in $\pi^{+} p \rightarrow \rho^{0} N^{*}$ at $3.65 \mathrm{GeV} / c$, and accounted for by pion exchange plus absorption. But for these reactions the model predicts absolute cross sections considerably larger than observed in the momentum range 3-4 GeV/c, a difficulty inherent in the distortedwave Born approximation when the coupling constants become large.

(8) The most clear-cut distinction between the absorptive model and the peripheral model with form factors occurs in the decay correlations. From the viewpoint of the latter model the experimental density matrices do not, by and large, correspond to the exchange of a single particle of definite spin. In contrast, the modifications produced by absorption explain the experimental decay correlations in many instances without the need for a mixture of different exchanges. For example, the theory presented here and in I predicts correctly the density-matrix elements for $\pi p \rightarrow \rho p$ assuming only $\pi$ exchange. Were the model without absorption adjusted to fit the data at, say, $4 \mathrm{GeV} / c$ by an admixture of vector exchange with suitable form factors, it would fail to reproduce the differential cross section at $8 \mathrm{GeV} / c$. Even more convincing is the large value of $\rho_{00}$ observed in $\omega$ production at $3.25 \mathrm{GeV} / c$ and in the reaction ${ }^{47} \pi^{+} p \rightarrow \omega N$ : in the $\rho$-exchange model without absorption this matrix element vanishes. Similarly, the combined correlation effects in $\pi^{+} p \rightarrow \rho N^{*}$ find a natural explanation in the present model, but necessitate more complicated exchanges in the peripheral model with form factors.

(9) Large, if not total, absorption in the lowest partial waves of the final state appears to be necessary. ${ }^{60}$

(10) The shortcomings and ambiguities of the model preclude reliable determinations of coupling constants. Apart from the questionable assumptions underlying the model, important ambiguities stem from the completely unknown final-state scattering and the simplified description of the initial- and final-state elastic scatter-

${ }^{60}$ In view of this, models wherein $C_{-} \leq 0.5$ [M. Baker and R. Blankenbecler, Phys. Rev. 128, 415 (1962); M. Ross and G. L. Shaw, Ref. 4] cannot account quantitatively for the data. 


\title{
B 446 J ACKSON, DONOHUE, GOTTFRIED, KEYSER, AND SVENSSON
}

ing in terms of a spin-independent, purely imaginary amplitude. The incorrect energy dependence of vectormeson-exchange amplitudes is the most important shortcoming, and prevents a meaningful determination of vector-meson coupling constants. ${ }^{61}$ Another difficulty is the violation (or near violation) of the unitarity bound in some reactions (e.g., $K p \rightarrow K^{*} N^{*}$ ).

${ }^{61} \mathrm{~A}$ further illustration of this remark is provided by the unsuccessful calculations of $\pi-N$ and $\bar{K}-N$ charge exchange scattering [V. Barger and M. Ebel, Phys. Rev. 138, B1148 (1965)]. While one may attribute this failure to the vector-meson-exchange mechanism, it is probably also due to the inapplicability of the model to processes that are, in essence, elastic. From the viewpoint of unitary symmetry $\left(\mathrm{SU}_{3}\right)$, calculations of associated ropduction and reactions such as $\bar{p} p \rightarrow \bar{\Lambda} \Lambda$ are open to the same doubts. Of course, within the framework of $S U_{6}$ all the processes considered in this paper are elastic scatterings!
(11) The physical assumptions of the model (and also the mathematical approximations) are most reliable at small production angles and at energies such that many partial waves participate in the reaction. Precise data in this domain would allow definitive tests of theory.

\section{ACKNOWLEDGMENTS}

We are grateful for the continued interest and support of Professor L. Van Hove and Professor V. F. Weisskopf at CERN, and two of us (K.G. and J.D.J.) express our thanks for the kind hospitality extended to us during our stay at CERN where this work began. We also wish to thank the many experimenters who communicated their results before publication. The references and footnotes bear witness to their generosity.

\section{Mass Relations of $S U_{3}$ in a Soluble $\omega-\phi$ Mixing Model ${ }^{*}$}

\author{
Herbert V. R. Pietschmann $\dagger$ \\ Department of Physics, University of Virginia, Charlottesville, Virginia
}

(Received 5 March 1965)

\begin{abstract}
A soluble model of $S U_{3}$-invariant meson-vecton interactions is investigated. $\omega$ - $\phi$ mixing is introduced as a soluble, symmetry-breaking interaction. Small form-factor effects in this interaction are also treated. Mass renormalization is carried out to study higher order effects in the Gell-Mann-Okubo formula for mesons. Two new mass formulas for vectons and an equation relating vecton masses to meson masses is derived. Finally, relations between bare coupling constants are studied. The agreement with experiment is generally good.
\end{abstract}

\section{INTRODUCTION}

$I^{1}$ $\mathrm{N}$ view of the apparent success of the octet version of unitary symmetry $\left(\mathrm{SU}_{3}\right)^{1-3}$ it is of increasing importance to obtain some understanding for the surprising validity of the Gell-Mann-Okubo mass formula (GMO formula). ${ }^{2,4}$ It has been derived in first-order perturbation theory with respect to the symmetrybreaking interaction, and nothing is known so far about the behavior of higher order contributions. We therefore set up a model which can be solved exactly and derive mass relations from it. In order to do this, we have to decide what kind of symmetry-breaking interaction we will choose. There are, of course, several ways to introduce symmetry-breaking effects. For reasons, specified below, we will choose $\omega-\phi$ mixing $^{5-7}$ for our

\footnotetext{
* Supported in part by the U. S. Atomic Energy Commission. $\dagger$ On leave from the University of Vienna, Vienna, Austria.

1 J. Wess, Nuovo Cimento 15, 52 (1960).

2 M. Gell-Mann, Phys. Rev. 125, 1067 (1962).

${ }^{3}$ Y. Ne'eman, Nucl. Phys. 26, 222 (1961).

4 S. Okubo, Progr. Theoret. Phys. (Kyoto) 27, 949 (1962); 28, 64 (1962).

5 J. J. Sakurai, Phys. Rev. 132, 434 (1963).

${ }^{6}$ S. L. Glashow, Phys. Rev. Letters 11, 48 (1963).

${ }^{7}$ S. Okubo, Phys. Letters 5, 165 (1963).
}

model: (1) Since we do not know the exact type of the basic symmetry-breaking interaction, $\omega-\phi$ mixing will serve as a good phenomenological description. Even if the fundamental interaction is of a completely different nature, $\omega-\phi$ mixing has to emerge as an "effective interaction." (2) A more technical reason for choosing $\omega-\phi$ mixing is that it is bilinear in the field operators and can thus be solved exactly.

The model we propose is an $\mathrm{SU}_{3}$-invariant version of the Zachariasen-Thirring ${ }^{8-11}$ model with $\omega-\phi$ mixing. In its Lagrangian version, the Zachariasen-Thirring model is nothing but a restriction on the type of Feynman graphs which have to be summed up. Our results can therefore be viewed either as the exact solution of a Zachariasen-Thirring model or as the chain approximation to a full-fledged theory. In any case they are valid to every order in the symmetry-breaking interaction.

We will study the interactions of (pseudoscalar) mesons and vector mesons ("vectons"). Their propaga-

\footnotetext{
${ }^{8}$ F. Zachariasen, Phys. Rev. 121, 1851 (1961).

${ }^{9}$ W. Thirring, Phys. Rev. 126, 1209 (1962).

${ }^{10} \mathrm{~W}$. Thirring, Nuovo Cimento 23, 1064 (1962).

${ }^{11} \mathrm{~W}$. Thirring, in Theoretical Physics (International Atomic Energy Agency, Vienna, 1963), p. 451.
} 